\title{
PERK is required at the ER-mitochondrial contact sites to convey apoptosis after ROS-based ER stress
}

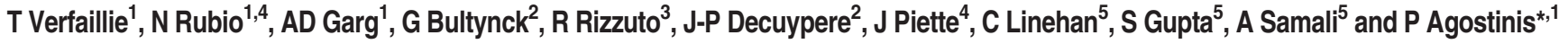

Endoplasmic reticulum stress is emerging as an important modulator of different pathologies and as a mechanism contributing to cancer cell death in response to therapeutic agents. In several instances, oxidative stress and the onset of endoplasmic reticulum (ER) stress occur together; yet, the molecular events linking reactive oxygen species (ROS) to ER stress-mediated apoptosis are currently unknown. Here, we show that PERK (RNA-dependent protein kinase (PKR)-like ER kinase), a key ER stress sensor of the unfolded protein response, is uniquely enriched at the mitochondria-associated ER membranes (MAMs). PERK ${ }^{-I-}$ cells display disturbed ER morphology and $\mathrm{Ca}^{2+}$ signaling as well as significantly weaker ER-mitochondria contact sites. Re-expression of a kinase-dead PERK mutant but not the cytoplasmic deletion mutant of PERK in PERK ${ }^{-l-}$ cells re-establishes ER-mitochondria juxtapositions and mitochondrial sensitization to ROS-mediated stress. In contrast to the canonical ER stressor thapsigargin, during ROS-mediated ER stress, PERK contributes to apoptosis twofold by sustaining the levels of pro-apoptotic C/EBP homologous protein (CHOP) and by facilitating the propagation of ROS signals between the ER and mitochondria through its tethering function. Hence, this study reveals an unprecedented role of PERK as a MAMs component required to maintain the ER-mitochondria juxtapositions and propel ROS-mediated mitochondrial apoptosis. Furthermore, it suggests that loss of PERK may cause defects in cell death sensitivity in pathological conditions linked to ROS-mediated ER stress.

Cell Death and Differentiation (2012) 19, 1880-1891; doi:10.1038/cdd.2012.74; published online 15 June 2012

The endoplasmic reticulum (ER) constitutes a specialized organelle involved in crucial cellular functions, including protein folding and $\mathrm{Ca}^{2+}$ storage/signaling. Alterations in the ER folding environment cause the accumulation of misfolded proteins in the ER lumen, leading to ER stress. ${ }^{1}$ To restore ER homeostasis, cells activate the unfolded protein response (UPR), a signaling pathway governed by three major ER stress sensors; PKR-like ER kinase (PERK), inositol requiring enzyme 1 (IRE1) and activating transcription factor 6 (ATF6). Together, these signaling pathways coordinate a temporal shut down in protein translation and induce a complex program of gene transcription to restore the folding capacity of the ER. However, when chronic or too severe, ER stress will engage apoptosis. ${ }^{2,3}$

Recently, reactive oxygen species (ROS) have emerged as crucial regulators of ER function and UPR activation and in several diseased conditions, ER stress and increased ROS production occur concurrently. ${ }^{4}$ Although our appreciation of the close association between oxidative stress and ER stress is increasing, the molecular events linking ROS to components of the ER stress machinery are still obscure. Moreover, how ROS modulate the ER-mitochondria cross-talk during apoptosis evoked by ER stress ${ }^{5,6}$ has not been fully investigated.

A paradigm of ROS-based anticancer modalities is photodynamic therapy, which exploits the combination of visible light, $\mathrm{O}_{2}$ and a light-absorbing molecule (i.e., photosensitizer) to produce detrimental doses of ROS in tumor tissue, leading to its ablation. ${ }^{7}$ In previous studies, we found that ROS produced by the light activation of hypericin, an ER membrane-localized photosensitizer, cause a rapid depletion of the ER $\mathrm{Ca}^{2+}$ stores, ultimately leading to mitochondrial dysfunction and apoptotic cell death. ${ }^{8}$ This rapid and selective burst in ROS generation at the ER, which we dubbed as 'photo-oxidative ER stress' (phox-ER stress), ${ }^{9}$ is also associated with the induction of a cluster of UPR genes, suggesting the functional propagation of an ER stress response. ${ }^{10}$ However, to what extent the activation of a specific component of the UPR signaling determines cellular fate following phox-ER stress remains to be clarified. Furthermore, we reasoned that this ROS-based paradigm could be extremely helpful in elucidating the still undefined molecular

\footnotetext{
${ }^{1}$ Laboratory of Cell Death Research and Therapy, Department of Cellular and Molecular Medicine, KU Leuven, Leuven, Belgium; ${ }^{2}$ Laboratory of Molecular and Cellular Signaling, Department of Cellular and Molecular Medicine, KU Leuven, Leuven, Belgium; ${ }^{3}$ Department of Biomedical Sciences, Neuroscience Institute of the National Research Council (CNR), University of Padova, Padova, Italy; ${ }^{4}$ Laboratory of Virology \& Immunology, GIGA-Research B34, University of Liège, Liège, Belgium and ${ }^{5}$ Apoptosis Research Centre, School of Natural Sciences, National University of Ireland, Galway, Ireland

${ }^{*}$ Corresponding author: P Agostinis, Laboratory of Cell Death Research and Therapy, Department of Cellular and Molecular Medicine, KU Leuven, Campus Gasthuisberg, O\&N1, Herestraat 49, Box 901, Leuven 3000, Belgium. Tel: +32 16 330650; Fax: +32 16 330735; E-mail: patrizia.agostinis@med.kuleuven.be Keywords: PERK; mitochondria-associated membranes; ROS; ER stress; apoptosis; photodynamic therapy

Abbreviations: $\mathrm{CHOP}, \mathrm{C} / \mathrm{EBP}$ homologous protein; elF2 $\alpha$, eukaryotic initiation factor-2 $\alpha$; ER, endoplasmic reticulum; GRP78, glucose regulated protein 78; $I_{3} R$, inositol 1,4,5-trisphosphate receptor; IRE1, inositol requiring enzyme 1; JNK, c-Jun N-terminal kinase; MAM, mitochondria-associated ER membrane; MEF, murine embryonic fibroblast; MFN2, mitofusin 2; PERK, RNA-dependent protein kinase (PKR)-like ER kinase; Phox, photo-oxidative; ROS, reactive oxygen species; SERCA, sarco/endoplasmic reticulum $\mathrm{Ca}^{2+}$ ATPase; TG, thapsigargin; UPR, unfolded protein response

Received 14.9.11; revised 01.5.12; accepted 07.5.12; Edited by SH Kaufmann; published online 15.6.12
} 
pathways linking ER stress to mitochondrial apoptosis in cells responding to oxidative stress.

Here, we reveal an unprecedented role for the ER stress sensor PERK, as an essential component of the mitochondria-associated ER membranes (MAMs) that establish a physical and functional connection between the ER and the mitochondria. ${ }^{11}$ We show that besides an aberrant ER morphology and $\mathrm{Ca}^{2+}$ signaling, $\mathrm{PERK}^{-1-}$ cells also display weaker contact sites between ER and mitochondria. Consequently, PERK-but not IRE1 ablation-reduces apoptosis following insults requiring the transfer of ROS-mediated signals between the ER and mitochondria. Collectively, our data reveal that besides its canonical ER stress sensor function, PERK serves as a structural tether at the ER-mitochondria interface regulating inter-organellar crosstalk in ROS-induced cell death.

\section{Results}

phox-ER stress induces the functional activation of the UPR. Based on our recent data indicating that in cancer cells responding to phox-ER stress, PERK-dependent pathways are predominantly induced ${ }^{10}$ and coordinate the induction of tumor-specific immune responses, ${ }^{9}$ we decided to unravel the role of this ER stress sensor in ROS signaling.

We used PERK ${ }^{+1+}$ and PERK $^{-1-}$ murine embryonic fibroblasts (MEFs), which displayed similar hypericin uptake and subcellular localization as well as ROS generation after excitation/irradiation (Supplementary Figures $1 \mathrm{~A}-\mathrm{C}$ ), thus allowing a direct comparison of phox-ER stress induced responses.

Phox-ER stress was associated with the morphological hallmarks of ER stress indicated by the expansion of the ER compartment (Figure 1a) and induction of a number of welldefined UPR target genes (Figures $1 b-d$ ). The qRT-PCR analysis confirmed that known PERK-regulated genes, like glucose regulated protein 78 (GRP78) and C/EBP homologous protein (CHOP), were attenuated in $\mathrm{PERK}^{-1-}$ cells (Figures $1 \mathrm{~b}-\mathrm{d}$ ). Consistent with the reported requirement of ATF6 $\alpha$ for transcriptional regulation of the majority of UPR chaperones and as a heterodimer with XBP1 for ERADrelated targets, ${ }^{12,13}$ the induction of several chaperones/ enzymes (GRP94, ERp72, p5, p58IPK, ERO1L, ERO1Lb) and ERAD components (HERP, Hrd1, Derlin-2, EDEM1) was either not affected or even heightened, ${ }^{14}$ in phox-treated PERK $^{-1-}$ cells (Figures $1 \mathrm{~b}$ and $\mathrm{c}$ ).

In PERK ${ }^{+/+}$cells, phox-ER stress led to PERK activation, following an initial partial drop in PERK levels as previously observed with sarco/endoplasmic reticulum $\mathrm{Ca}^{2}+$ ATPase $\left(\right.$ SERCA) ${ }^{8}$ eukaryotic initiation factor- $2 \alpha$ (elF2 $\alpha$ ) phosphorylation (elF2 $\alpha-P$ ), GRP78 and CHOP accumulation (Figures $2 a$ and $b)$ along with induction of the spliced form (XBP1s) of the IRE1 target XBP1 (Figures 2c-f). In line with the qRT-PCR results, PERK ablation mitigated GRP78 increase, reduced CHOP protein levels and resulted in a somewhat sustained activation of the IRE1 branch of the UPR, as indicated by

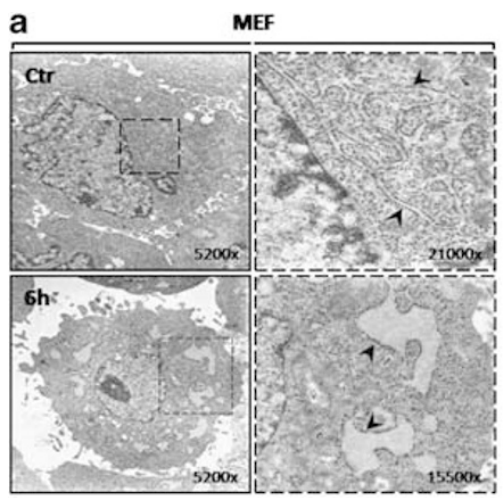

b
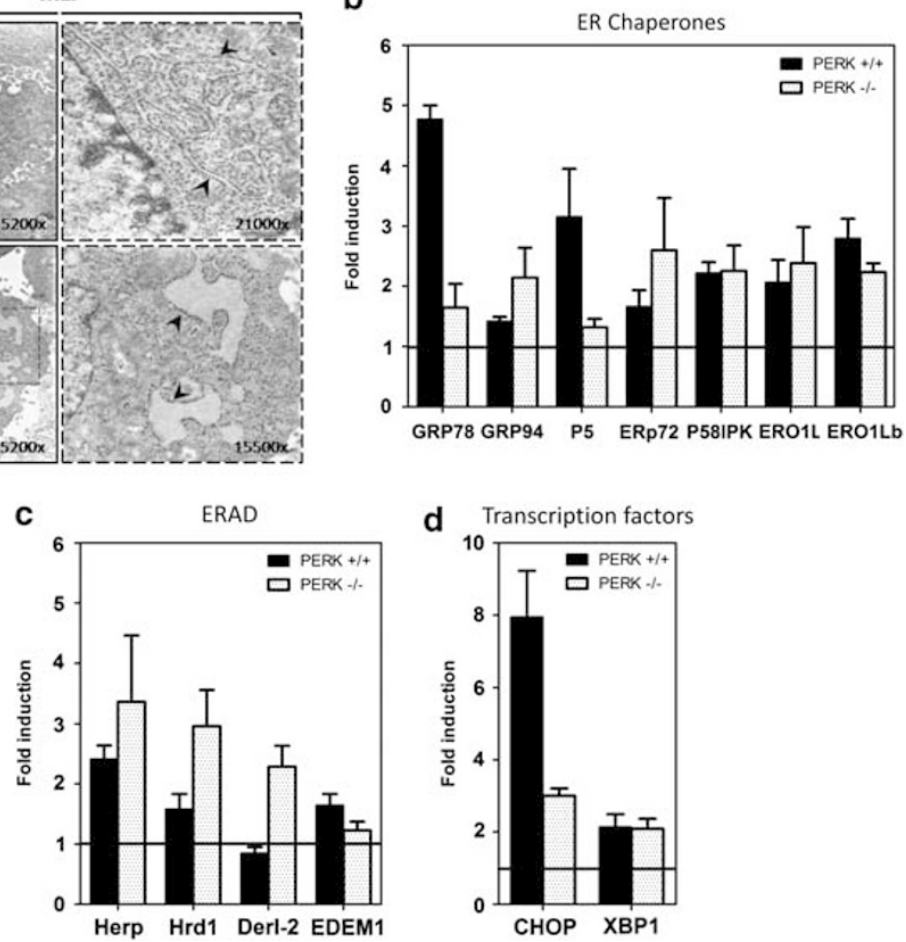

Figure 1 Photo-oxidative treatment with hypericin causes ER stress and subsequent induction of multiple UPR target genes. (a) TEM analysis of MEFs before and $6 \mathrm{~h}$ after photo-oxidative (phox) treatment. Enlarged on the right is the perinuclear rough ER region indicated in the left images. Arrows indicate ER lamellae before and after treatment. (b-d) Total RNA was extracted from control and phox-treated PERK ${ }^{+1+}$ and PERK ${ }^{-1-}$ MEFs, $6 \mathrm{~h}$ after irradiation. mRNA levels of the indicated genes, grouped into (b) ER chaperones, (c) ERAD proteins and (d) UPR-related transcription factors, were quantified by qRT-PCR, normalized against GAPDH and expressed as fold change versus control. Graphs represent mean \pm S.E.M. of three independent experiments performed in duplicate 
a

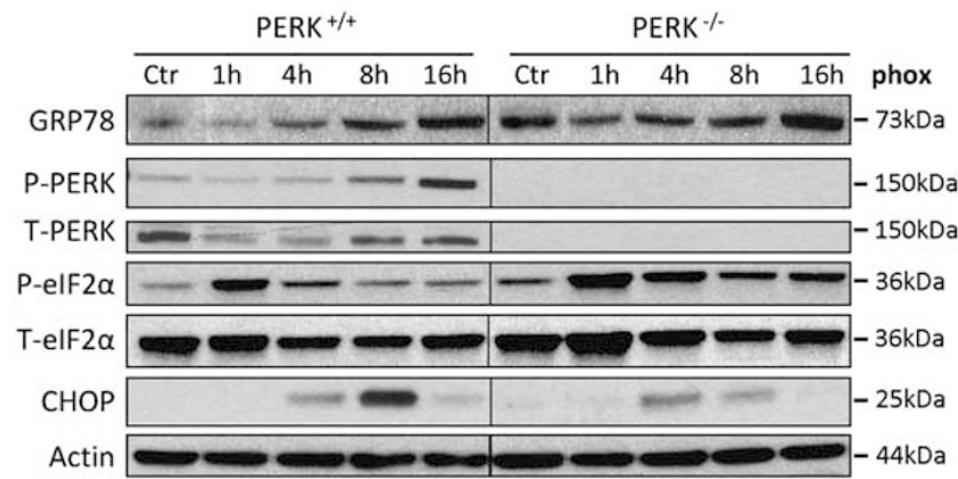

GRP78

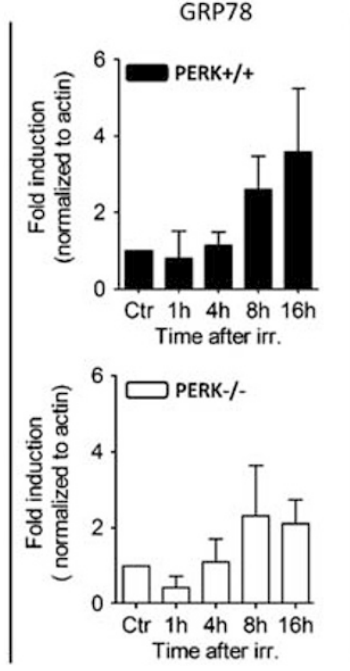

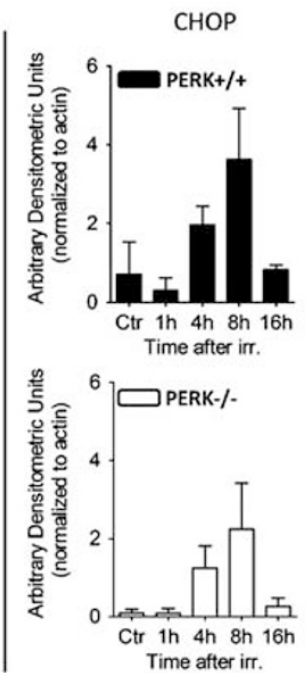

c

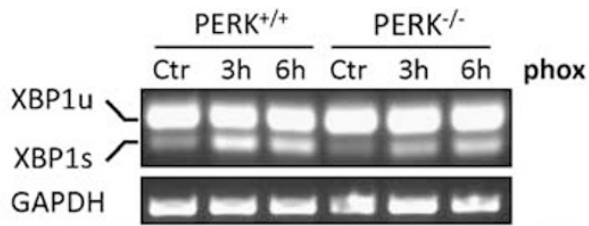

d

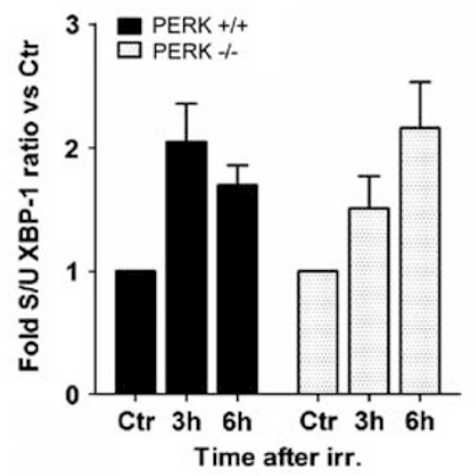

e
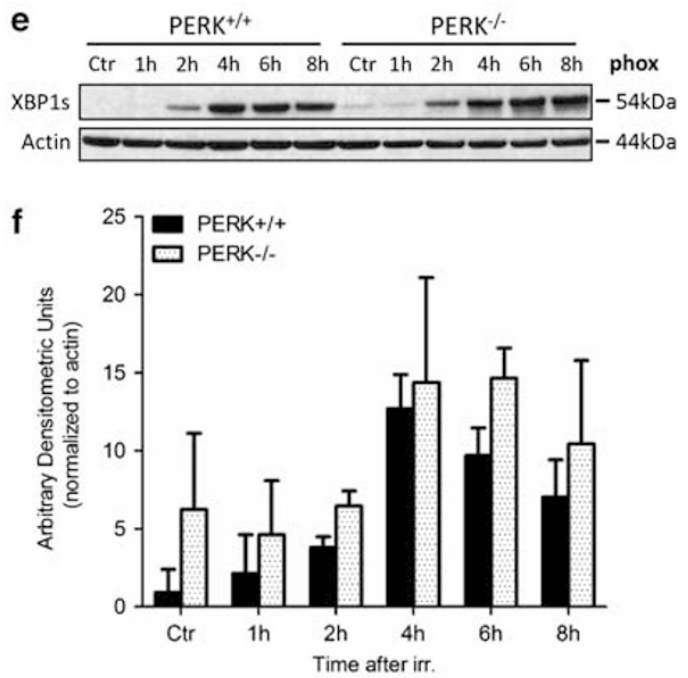

Figure 2 Activation of UPR signaling following phox-induced ER stress. (a) After phox treatment of PERK ${ }^{+1+}$ and PERK ${ }^{-1-}$ MEFs, whole cell lysates were made at the indicated time points and immunoblotted for the indicated proteins. A typical immunoblot from the same membrane is shown. (b) Densitometric analysis of immunoblots from elF2 $\alpha$ phosphorylation, GRP78 and CHOP induction after phox treatment. Graphs represent mean \pm S.D. of three independent experiments. (c) After phox treatment of PERK $^{+1+}$ and PERK ${ }^{-1-}$ MEFs, total RNA was extracted at the indicated time points. RT-PCR analysis was performed to simultaneously detect the spliced (s) and unspliced (u) forms of XBP1 and GAPDH. XBP1u $=289 \mathrm{bp}, \mathrm{XBP} 1 \mathrm{~s}=263 \mathrm{bp}$. (d) Densitometric analysis of XBP1 splicing as performed in (c). The ratio of spliced XBP1(s)/ unspliced XBP1(u) was calculated and expressed as fold change versus control. Graph represents mean \pm S.D. of three independent experiments performed in duplicate. (e) After phox treatment of PERK ${ }^{+1+}$ and PERK ${ }^{-1}$ MEFs, whole cell lysates were made at the indicated time points and immunoblotted for spliced XBP1 and actin as loading control. (f) Densitometric analysis of immunoblots for spliced XBP1 after phox treatment. Graphs represent mean \pm S.D. of three independent experiments

the steady accumulation of the active transcription factor XBP1s at later time points following phox treatment (Figures $2 a-f)$. elF2 $\alpha$-P was not attenuated in phox-treated PERK ${ }^{-1-}$ cells, thus implicating the possible involvement of other elF2 $\alpha$ kinases.

Altogether, these results underpin the functional activation of the UPR after phox-ER stress.

Lack of PERK protects against ROS-induced ER stressmediated apoptosis. In response to phox treatment, PERK is required to mount sustained levels of pro-apoptotic $\mathrm{CHOP}$ (Figure 2a), suggesting a role for PERK in relaying apoptotic cell death. Consistent with the reduced CHOP induction, caspase cleavage in PERK ${ }^{-1-}$ cells was clearly blunted after phox-ER stress (Figure 3a) and these cells were significantly more resistant to phox-mediated cell death compared with their WT counterparts (Figure 3b). This was not the result of a clonal artifact since both murine colon carcinoma CT26 and human breast cancer MDA-MB468 cell lines stably transduced with shRNA-PERK, which reduced PERK expression (Supplementary Figure 2A), were also more resistant to phox-ER stress induced caspase activation (Supplementary Figure 2B) and overall apoptotic cell death (Supplementary Figures 2C-E) as compared with their counterparts transduced with a scrambled sequence. 

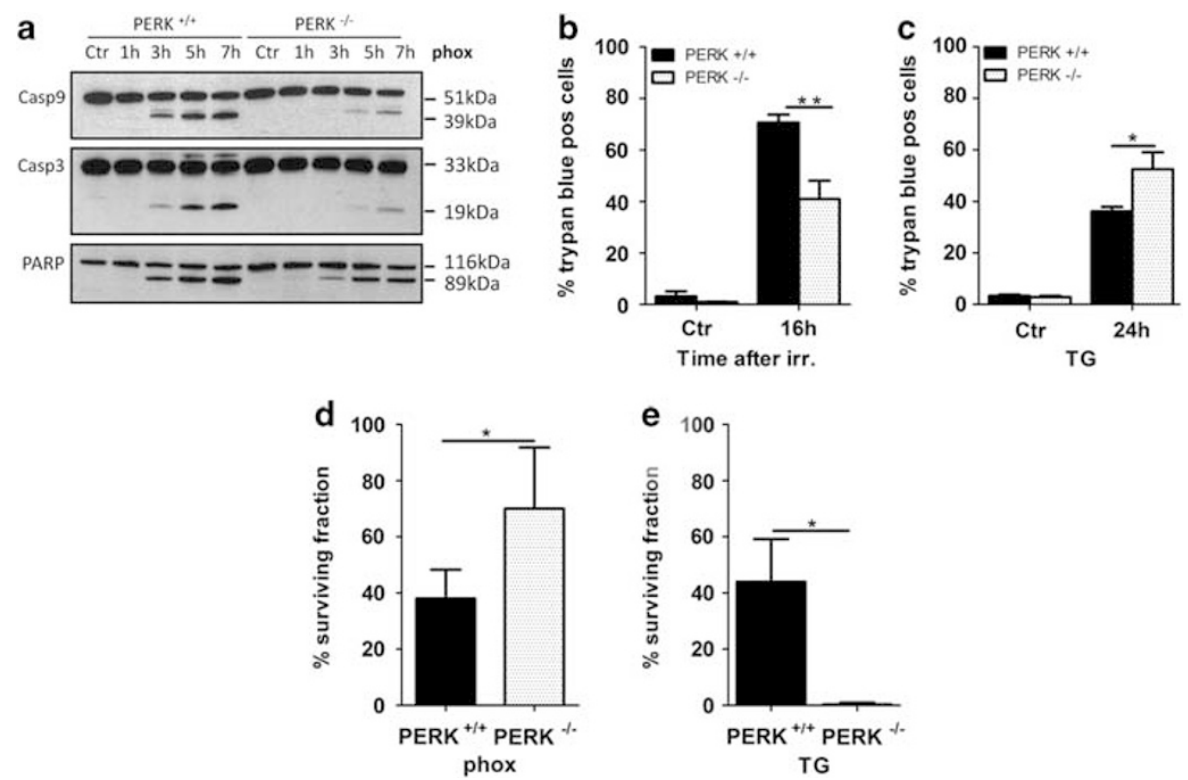

Figure 3 PERK deficiency protects cells against phox-ER stress induced but not TG induced cell death. (a) After phox treatment of PERK ${ }^{+1+}$ and PERK ${ }^{-1-}$ MEFs, whole cell lysates were made at the indicated time points and immunoblotted for the indicated proteins. (b and $\mathbf{c}$ ) Effect of PERK deficiency on cell death after phox treatment (b) or TG $(2 \mu \mathrm{M}, \mathbf{c})$. Percentage of trypan blue-positive cells (dead cells) at the indicated time points is shown. Graphs represent the mean \pm S.D. of three independent experiments performed in duplicate. (d and e) Effect of PERK deficiency on colony formation after phox treatment (d) or TG $(2 \mu \mathrm{M}$, e). Percentage of surviving fraction was determined 10 days after replating of control and treated (immediately after phox treatment or $16 \mathrm{~h}$ after TG) KRAS transformed PERK ${ }^{+1+}$ and PERK ${ }^{-1-}$ MEFs by counting colonies consisting of at least 50 cells and comparing treated versus non-treated conditions. Graphs represent the mean \pm S.D. of three independent experiments performed in triplicate. $\left(^{*}\right)$ and $\left(^{* *}\right)$ indicate $P<0.05$ and $P<0.005$, respectively

PERK deficiency also mitigated caspase signaling and cell death after $\mathrm{H}_{2} \mathrm{O}_{2}(2 \mathrm{mM})$ (Supplementary Figures $\left.2 \mathrm{~F}-\mathrm{H}\right)$, a broad spectrum pro-oxidant molecule inducing $\mathrm{Ca}^{2+}$ release from agonist-sensitive stores through redox modulation of both inositol 1,4,5-trisphosphosphate $\left(\mathrm{IP}_{3}\right)$ receptors $\left(\mathrm{IP}_{3} \mathrm{Rs}\right)$ and SERCA,${ }^{15}$ ultimately causing ER stress. ${ }^{16}$ The absence of PERK moreover significantly increased the clonogenic capacity of KRAS-transduced PERK ${ }^{-1-}$ MEFs after phox-ER stress (Figure 3d).

In contrast, lack of PERK increased cell death (Figure 3c) and abolished clonogenic survival (Figure $3 e$ ) after treatment with thapsigargin (TG, an irreversible inhibitor of SERCA) causing the depletion of $\mathrm{ER} \mathrm{Ca}^{2+}$ stores. This is consistent with a reported protective role for the PERK-elF2 $\alpha$ axis in TGtreated cells. ${ }^{17}$

To consider the role of other UPR sensors, we examined IRE $1^{+/+}$and IRE1 $1^{-/-}$MEFs. Consistent with previous reports, ${ }^{18}$ IRE1-deficiency reduced C-Jun N-terminal kinase (JNK) and caspase signaling following TG treatment (Supplementary Figure 3A). In contrast, JNK activation and caspase signaling after phox-ER stress were not altered by IRE1 deficiency (Supplementary Figure 3B), thus arguing that ROS-mediated ER stress relies predominantly on the PERK branch of the UPR to propagate apoptosis, while IRE1 is dispensable.

PERK deficiency results in a fragmented ER morphology and disturbed $\mathrm{Ca}^{2+}$ signaling. Consistent with previous findings in secretory and muscle cells from $\mathrm{PERK}^{-1-}$ mice, ${ }^{19}$ fluorescent staining of the ER revealed a diffuse pattern in PERK ${ }^{+1+}$ MEFs while the ER from PERK $^{-1-}$
MEFs displayed a more 'fragmented' morphology (Supplementary Figure 4A).

Since this morphological defect was previously associated with aberrant $\mathrm{Ca}^{2+}$ signaling, ${ }^{19}$ we next analyzed $\mathrm{ER} \mathrm{Ca}^{2+}$ dynamics in Fura-2-loaded PERK ${ }^{+/+}$and PERK ${ }^{-/-}$MEFs treated with the $\mathrm{IP}_{3}$ generating agent ATP $(10 \mu \mathrm{M})$. EGTA was added to buffer all extracellular $\mathrm{Ca}^{2+}$ to assess only $\mathrm{Ca}^{2+}$ fluxes originating from intracellular stores.

Compared with $\mathrm{PERK}^{+/+} \mathrm{MEFs}, \mathrm{PERK}^{-1-}$ MEFs displayed severely blunted $\mathrm{Ca}^{2+}$ signals in response to ATP (Figure 4a). Similarly, we treated the cells with TG for which the resulting increase in cytosolic $\mathrm{Ca}^{2+}$ is an indirect assessment of the $\mathrm{ER} \mathrm{Ca}^{2+}$ store content. The $\mathrm{Ca}^{2+}$ signals provoked by TG were not significantly altered in $\mathrm{PERK}^{-1-}$ cells (Figure 4b), indicating that the $\mathrm{ER} \mathrm{Ca}^{2+}$ stores are properly loaded and cannot account for the differences in ATP-induced $\mathrm{Ca}^{2+}$ signaling in these cells. A similar analysis in IRE $1^{+/+}$and IRE $1^{-/-}$cells revealed neither significant differences in ER morphology (data not shown) nor in $\mathrm{Ca}^{2+}$ release kinetics (Supplementary Figures $3 \mathrm{C}$ and $\mathrm{D}$ ), indicating that these phenomena are specific for PERK.

The severely reduced ATP-induced $\mathrm{Ca}^{2+}$ signals in PERK $^{-1-}$ cells suggested that either $I P_{3} R$ activity is compromised or the fragmented ER morphology affects the diffusion of $\mathrm{IP}_{3}$, generated near the subplasmalemmal region following engagement of the purinergic P2Y receptors, to the ER-located IP ${ }_{3}$ Rs.

To test these possibilities, we first assessed $I P_{3} R$ mediated $\mathrm{Ca}^{2+}$ release independently from diffusion processes by performing a unidirectional ${ }^{45} \mathrm{Ca}^{2+}$-flux assay in plasma membrane-permeabilized cells in which the 

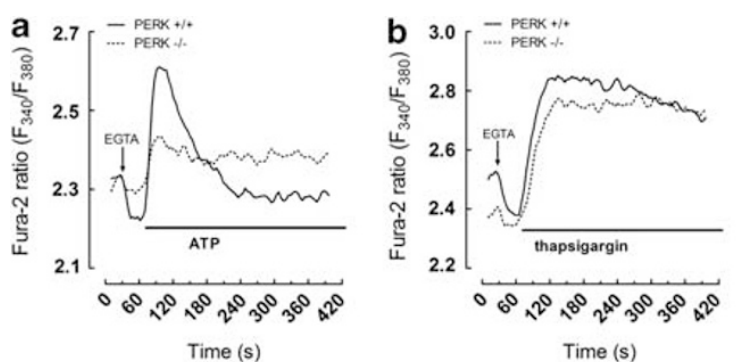

C

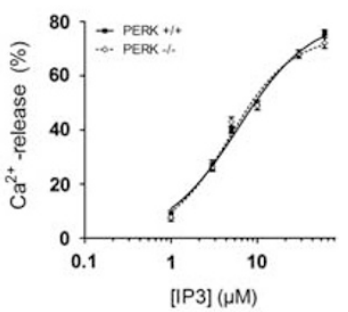

d

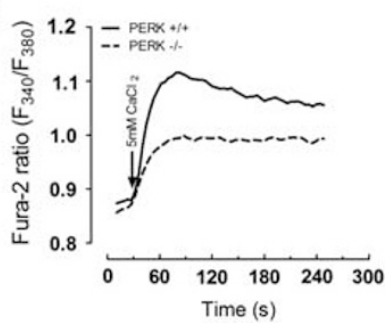

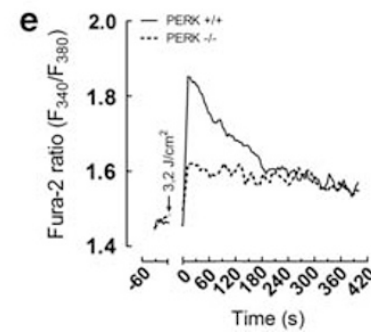

Figure 4 PERK-deficient MEFs display aberrant $\mathrm{Ca}^{2+}$ release in response to phox-ER stress. (a and $\left.\mathbf{b}\right)$ Analysis of cytosolic calcium release. Fura2-loaded $\mathrm{PERK}^{+1+}$ and PERK ${ }^{-1-}$ MEFs were incubated in modified $\mathrm{Ca}^{2+}$-free KREBS buffer and cytosolic $\mathrm{Ca}^{2+}$ signals were measured as $340 / 380$ fluorescence $(\mathrm{F})$ ratios. At the indicated time point (arrow), EGTA $(3 \mathrm{mM})$ was added. After $30 \mathrm{~s}$, cells were challenged with ATP $(10 \mu \mathrm{M})$ or TG $(1 \mu \mathrm{M})$. Representative traces are shown $(n=2)$. (c) ${ }^{45} \mathrm{Ca}^{2+}$-flux analysis comparing $\mathrm{IP}_{3} \mathrm{R}$ activity in permeabilized PERK ${ }^{+1+}$ and PERK ${ }^{-1-} \mathrm{MEFs}$, in which the ER is loaded to steady state with ${ }^{45} \mathrm{Ca}^{2+}$. Graph represents data obtained by normalizing the $\mathrm{IP}_{3}$-induced $\mathrm{Ca}^{2+}$ release to the A23187-releasable ${ }^{45} \mathrm{Ca}^{2+}$ in PERK ${ }^{+1+}$ and PERK ${ }^{-1-}$ cells. Results are presented as mean \pm S.D. $(n=3-4)$. (d) $\mathrm{Ca}^{2+}$-entry analysis in which Fura2-loaded PERK ${ }^{+1+}$ and PERK ${ }^{-1-}$ MEFs were pre-incubated for 30 min in modified $\mathrm{Ca}^{2+}$-free $\mathrm{KREBS}$ solution containing $2 \mathrm{mM} \mathrm{EGTA}$ and $1 \mu \mathrm{M}$ TG before recording of $\left[\mathrm{Ca}^{2+}\right]_{\text {cyt. }}$. At the indicated time (arrow), extracellular $\left[\mathrm{Ca}^{2+}\right]$ was raised to $5 \mathrm{mM}$ and store-operated $\mathrm{Ca}^{2+}$ influx was monitored. Representative traces are shown $(n=3)$. (e) PERK ${ }^{+1+}$ and PERK ${ }^{-1-}$ MEFs loaded with $1.25 \mu \mathrm{M}$ Fura-2AM and $1 \mu$ M hypericin were incubated in modified $\mathrm{Ca}^{2+}$-free KREBS buffer. Basal Fura-2 fluorescence ratio was measured for the indicated time period and cells were next irradiated (time $0,3.2 \mathrm{~J} / \mathrm{cm}^{2}$ ) on a low pressure sodium lamp. Changes in Fura-2 ratio were monitored immediately after irradiation. Representative traces are shown $(n=2)$

non-mitochondrial $\mathrm{Ca}^{2+}$ stores have been loaded to steady state with ${ }^{45} \mathrm{Ca}^{2+}$. We found that the responsiveness of the $I_{3} R$ to $I_{3}$ was almost identical between PERK ${ }^{+/+}$and $\mathrm{PERK}^{-1-}$ cells (Figure 4c; Supplementary Figure 4B), indicating proper intrinsic $\mathrm{IP}_{3} \mathrm{R}$ activity in $\mathrm{PERK}^{-/-}$cells.

Therefore, $\mathrm{PERK}^{-1-}$ cells may have defective $\mathrm{Ca}^{2+}$. signaling complexes connecting plasma membrane and $E R$, increasing the diffusion time between the sites of $\mathrm{IP}_{3}$ production and action. Consistent with this hypothesis, store-operated $\mathrm{Ca}^{2+}$ entry, which depends on the close proximity of the ER and the plasma membrane, ${ }^{20}$ is impaired in PERK $^{-1-}$ cells (Figure 4d).

We then analyzed the amplitude of $\mathrm{Ca}^{2+}$ signals in response to phox-ER stress. Compared with PERK ${ }^{+1+}$ cells, the PERK ${ }^{-I}$ cells displayed a reduction in the amplitude of $\mathrm{Ca}^{2+}$ signals induced by phox-ER stress (Figure $4 \mathrm{e}$ ). This is in striking contrast to the $\mathrm{Ca}^{2+}$ signals provoked by TG (Figure $4 b$ ). Since both agonists promote $\mathrm{Ca}^{2+}$ leak from the ER by inhibiting SERCA activity, ${ }^{8,15}$ this may indicate that phox-provoked $\mathrm{Ca}^{2+}$ signals involve $\mathrm{IP}_{3}$ signaling, which is likely the result of the activation of phospholipases, including phospholipase C. ${ }^{21,22}$

Hence, the fragmented ER morphology displayed by the $\mathrm{PERK}^{-1}$ cells disturbs agonist-induced $\mathrm{ER} \mathrm{Ca}^{2+}$ release and affects the amplitude of $\mathrm{Ca}^{2+}$ fluxes following phox treatment.

Lack of PERK protects mitochondria from ROS damage. We previously reported that BAX/BAK proteins at the mitochondria are indispensable for phox-mediated apoptosis. ${ }^{8}$ Since the PERK-deficient cells are protected against phox-mediated apoptosis, we next examined to what extent PERK deficiency could affect ROS-sensitive mitochondrial processes, inciting cell death.

We analyzed redox-mediated changes in cardiolipin, a phospholipid almost exclusively found in the inner mitochondrial membrane and whose oxidation facilitates leakage of cytochrome $c$ from mitochondria in different cell death paradigms. ${ }^{23}$

The percentage of PERK ${ }^{+/+}$cells with oxidized cardiolipin increased rapidly (as early as $1 \mathrm{~h}$ ) after phox-ER stress (Figure 5a). This process was concomitant with or even preceded cytochrome $c$ release in the cytosol (2-3h after treatment; Figure $5 \mathrm{~b}$ ), consistent with a proposed model wherein early, pre-MOMP (mitochondrial outer membrane permeabilization) cardiolipin oxidation provides a docking site for the recruitment/oligomerization of pro-apoptotic BAX to the mitochondrial membrane. ${ }^{24}$ Intriguingly, cells lacking PERK were significantly protected from this apical ROS-mediated event and its consequences, since cytosolic cytochrome c release (Figure 5b), as well as caspase-9 and caspase-3 processing were clearly delayed in these cells following phoxER stress (Figure 3b).

CHOP deficiency did not affect the extent of phox-ER stress induced 'pre-MOMP' cardiolipin oxidation, while it attenuated this event at later time points, coinciding with the abundant cytochrome $c$ release (i.e., post-MOMP; Figure $5 c$ ). These results strongly suggest that PERK regulates early ROSmediated mitochondrial events, which are detached from the induction of pro-apoptotic CHOP during UPR signaling. Additionally, while in the PERK ${ }^{+/+}$cells phox-ER stress caused a dose-dependent dissipation of the mitochondrial 
a

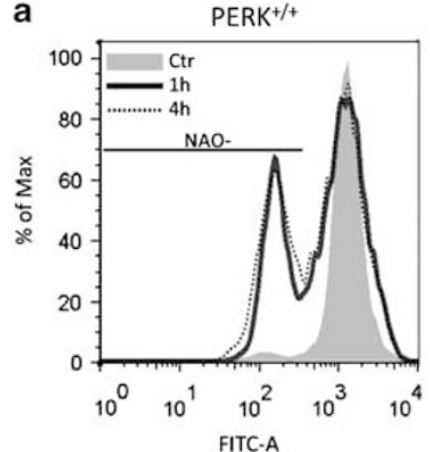

b

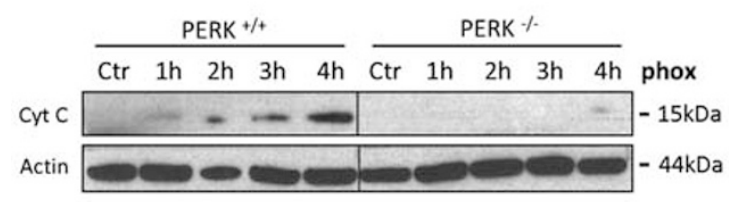

PERK $\%$

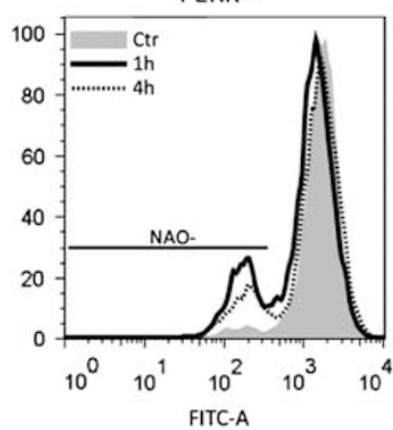

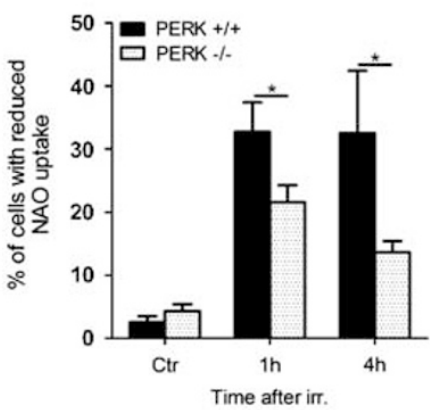

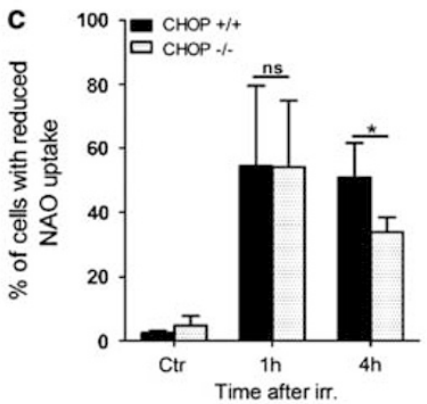

d
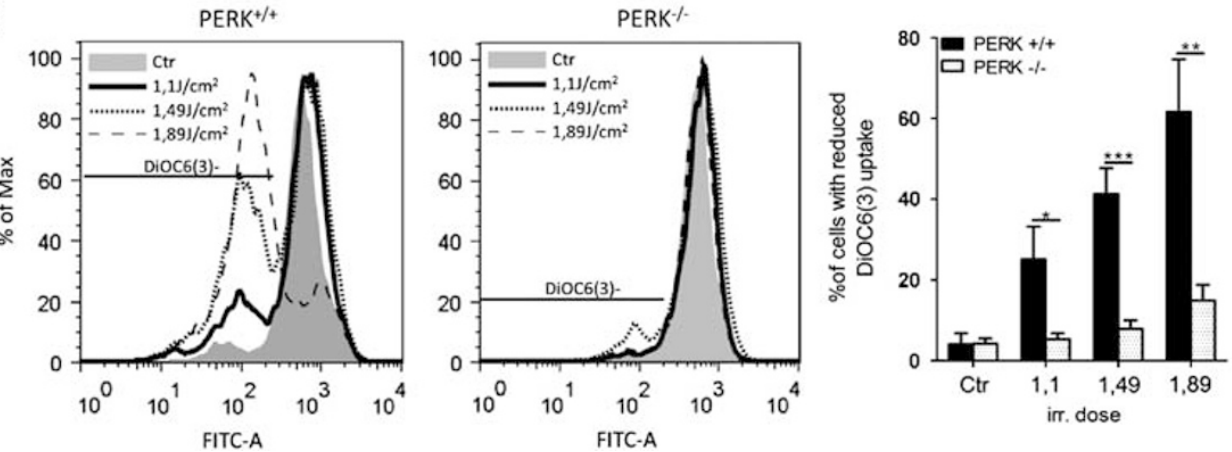

Figure 5 PERK deficiency protects cells from ROS-induced mitochondrial dysfunction. (a) Comparison of cardiolipin oxidation following phox-ER stress in PERK ${ }^{+/+}$and PERK ${ }^{-1}$ MEFs. Cells were left untreated or treated with phox-ER stress. At the indicated time points, cells were incubated with $10 \mathrm{nM}$ NAO and the percentage of cells with reduced cardiolipin binding (NAO -) was determined by flow cytometry. Left: histograms showing results for a representative experiment; right: graph represents the mean \pm S.D. of three independent experiments performed in duplicate. (b) Release of cytochrome $c$ (cyt $c$ ) following phox-ER stress in PERK ${ }^{+1+}$ and PERK ${ }^{-1-}$ MEFs. Cells were left untreated (Ctr) or phox treated. At the indicated time points, subcellular fractions were made for western blot analysis of cytochrome $c$ release in the cytosol. A representative western blot from three independent experiments is shown. Left and right parts are from the same membrane. (c) Comparison of cardiolipin oxidation following phox-ER stress in $\mathrm{CHOP}^{+1+}$ and $\mathrm{CHOP}^{-1-}$ MEFs. Cells were left untreated $(\mathrm{Ctr})$ or treated with phox-ER stress $\left(1.62 \mathrm{~J} / \mathrm{cm}^{2}\right)$. At the indicated time points, cells were incubated with $10 \mathrm{nM} \mathrm{NAO}$ and the percentage of cells with reduced cardiolipin binding (NAO) was determined by flow cytometry. Graph represents the mean \pm S.D. of three independent experiments performed in duplicate. (d) Dissipation of mitochondrial membrane potential $\left(\Delta \psi_{\mathrm{m}}\right)$ following phox-ER stress in PERK ${ }^{+1+}$ and PERK ${ }^{-1-}$ MEFs. Cells were left untreated (Ctr) or phox treated at the indicated irradiation dose. After $6 \mathrm{~h}$, cells were incubated with $2 \mathrm{nM}$ DiOC6(3) and the percentage of cells with reduced membrane potential (DiOC6(3) -) was determined by flow cytometry. Left: histograms showing results for a representative experiment. Right: graph represents the mean \pm S.D. of three independent experiments. ( $\left.{ }^{*}\right)$ indicates $\left.P<0.05,{ }^{* *}\right) P<0.005$ and $\left.{ }^{* * *}\right) P<0.001$

transmembrane potential $\left(\Delta \psi_{\mathrm{m}}\right)$ (Figure $5 \mathrm{~d}$ ), the PERKdeficient cells maintained their $\Delta \psi_{m}$ over a wide range of phox-ER stress doses.

In contrast, PERK deficiency increased the kinetics of $\Delta \psi_{\mathrm{m}}$ dissipation and cytochrome $c$ release as well as the onset of cardiolipin oxidation after TG treatment (Supplementary Figures 5A-C), highlighting the ER stressor-specific modulation of intrinsic apoptosis by PERK.

Thus, along with a fragmented ER and disturbed ER $\mathrm{Ca}^{2+}$ handling, mitochondria from $\mathrm{PERK}^{-1-}$ cells are protected from ROS-mediated events regulating apoptosis.
PERK deficiency disrupts the ER-mitochondria network. To elucidate how PERK protects mitochondria in response to phox-ER stress, we examined the ER-mitochondria interaction sites. It is well established that a subset of mitochondria resides in close contact with the ER at the opening of $\mathrm{IP}_{3}$-sensitive $\mathrm{Ca}^{2+}$ channels, enabling microdomains of high $\mathrm{Ca}^{2+}$ concentration and fine-tuning $\mathrm{Ca}^{2+}$ fluxes between these organelles. ${ }^{25}$ Moreover, rises in $\left[\mathrm{Ca}^{2+}\right]_{\text {cyt }}$ have been reported to induce a rapid, reversible and coordinated inhibition of ER and mitochondrial motility, ${ }^{26}$ which facilitates the recruitment of mitochondria to the region 
of the $\left[\mathrm{Ca}^{2+}\right]_{\text {cyt }}$ rise and their local $\mathrm{Ca}^{2+}$-buffering capacities. ${ }^{11}$

To examine differences in the ER-mitochondria interaction between PERK ${ }^{+/+}$and PERK ${ }^{-1-}$ cells, we colocalized the ER luminal protein calreticulin with the mitochondrial outer membrane protein TOM20 and subjected untreated cells to immunocytochemistry analysis.
Semiquantitative confocal microscopy analysis of ER-mitochondria juxtapositions unraveled that while mitochondria from PERK ${ }^{+1+}$ cells were clearly interweaved with the ER network, the mitochondria from PERK ${ }^{-1}$ cells colocalized with the ER to a significantly lower extent (Figure 6A, panels a and d). In the latter cells, the overlap between mitochondria and the ER was significantly decreased as compared with
A
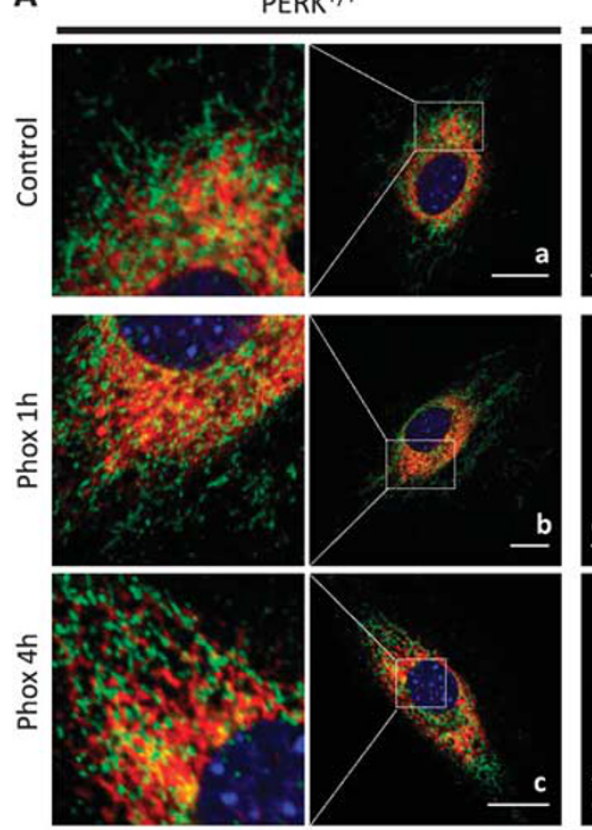

C

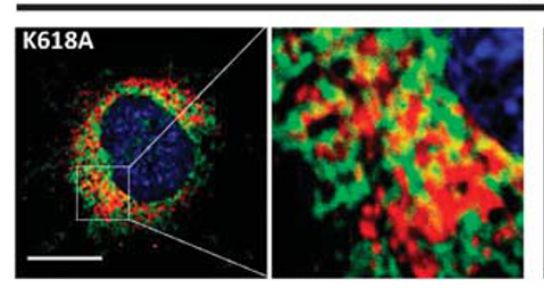

PERK - -

E

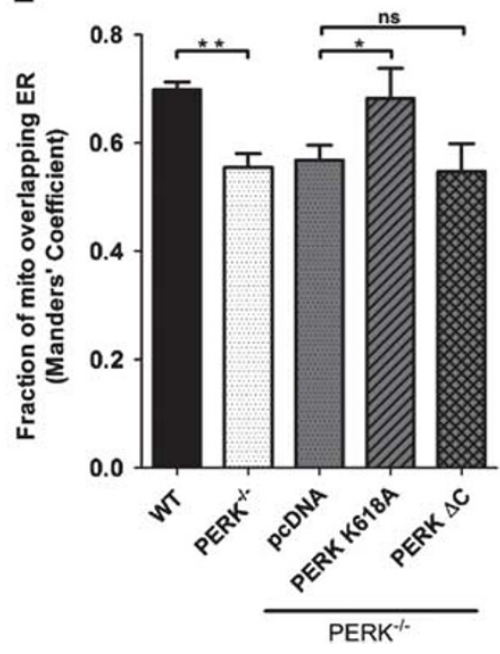

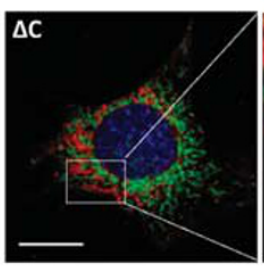

PERK \%
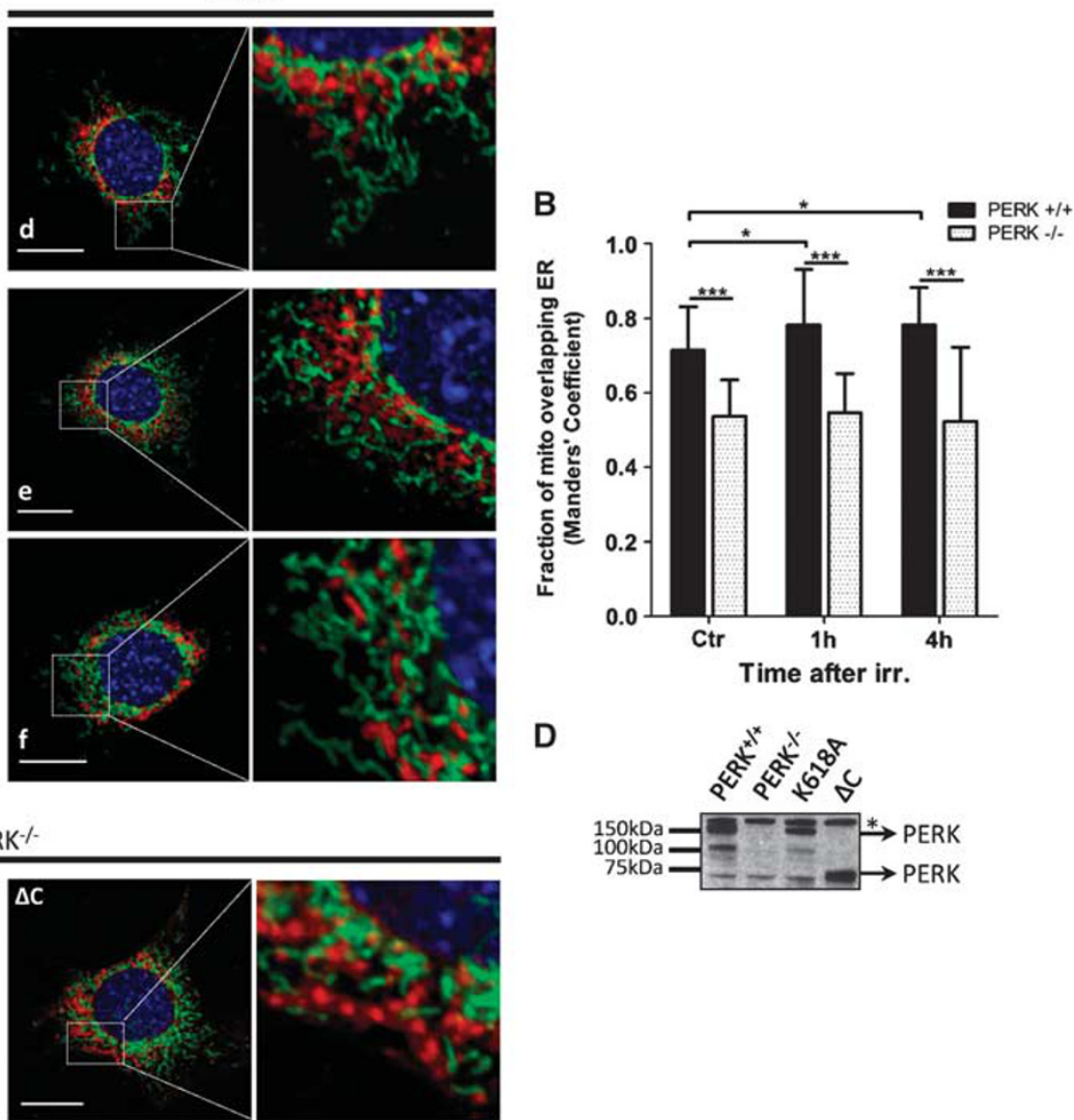

D

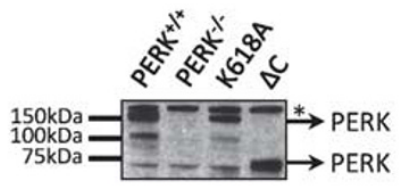

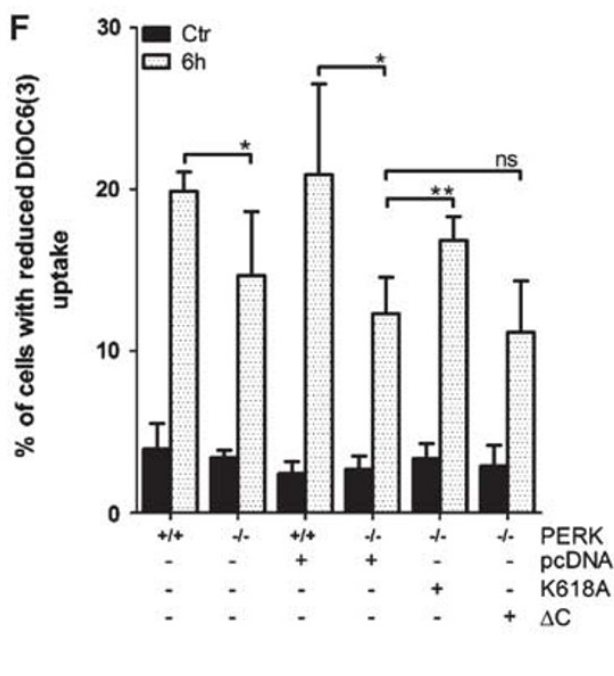


PERK $^{+1+}$ cells (Figure $6 \mathrm{~B}$ ). Since phox-ER stress entails both ER $\mathrm{Ca}^{2+}$ depletion ${ }^{8}$ and ROS signaling, which may cooperate in remodeling the ER-mitochondria network, we also analyzed the temporal evolution of ER-mitochondria dynamics. While in PERK ${ }^{+/+}$cells phox-ER stress induced a rapid (after $1 \mathrm{~h}$ ) recruitment of mitochondria to the ER and significantly increased the colocalization between these organelles (Figure 6A, panels b and c; Figure 6B), in $\mathrm{PERK}^{-1-}$ cells the separation of the mitochondria from the ER network persisted in response to phox-ER stress (Figure 6A, panels e and f; Figure 6B). These observations suggest a role for PERK in the modulation of the ERmitochondria network, which is tightened in response to phox-ER stress.

To determine whether the kinase activity of PERK is required to maintain these contact sites, we transfected $\mathrm{PERK}^{-1-}$ cells with a kinase-dead (K618A) mutant ${ }^{27}$ of PERK (Figure 6D). Expression of this PERK mutant restored the level of ER-mitochondria colocalization to that of PERK ${ }^{+1+}$ cells (Figure $6 \mathrm{C}$, left panel Figure 6E). Conversely, the expression of a mutant lacking the cytoplasmic domain of PERK (Figure $6 \mathrm{D}$ ), that is, PERK- $\Delta$ C, failed to re-establish the ER-mitochondria contact sites (Figure 6C, right panel; Figure $6 \mathrm{E}$ ). This indicates that while the cytoplasmic domain is essential, PERK activity is dispensable for maintaining the ER-mitochondria juxtapositions.

Notably, reconstitution of the kinase-dead PERK partially restored phox-mediated $\Delta \psi_{\mathrm{m}}$ loss, whereas expression of PERK- $\Delta C$ lacking the cytoplasmic domain required for ER-mitochondria tethering, failed to do so (Figure 6F). Since both PERK mutants lack the PERK-mediated UPR function, yet only the kinase-dead PERK mutant restores, at least partially, the $\Delta \psi_{\mathrm{m}}$ loss following phox-ER stress, these results underpin a novel function of PERK beyond the UPR.

The transient transfection of these PERK mutants, however, failed to re-establish proper ER morphology, which remained highly fragmented as observed in the PERK ${ }^{-1-}$ cells (Supplementary Figure 6A) and therefore could not rescue the defects in $\mathrm{Ca}^{2+}$ entry observed in $\mathrm{PERK}^{-1-}$ cells either (Supplementary Figures 6B and C).

In conclusion, these observations indicate that PERK deficiency/lack of the cytoplasmic domain deranges $\mathrm{Ca}^{2+}$ signaling and curtails the ER-mitochondria connections. Moreover, this defect appears to protect mitochondria from ROS-mediated ER stress.
PERK is a component of the MAMs. The results described above highlighted a profound morphological defect affecting the maintenance of the ER-mitochondria connection in cells lacking PERK. These specialized regions are called mitochondria-associated ER membranes or MAMs. ${ }^{11}$ Currently identified molecular components of the MAMs include the $\mathrm{IP}_{3} \mathrm{R} 3$, the SigmaR1 receptor, the voltage-dependent anion channel type 1 (VDAC-1), chaperones like calnexin, and mitofusin 2 (MFN2), a mitochondrial dynamin-like GTPase tethering mitochondria to the ER. ${ }^{11,28}$ Thus, we questioned whether PERK was a novel component of the MAMs.

We investigated the intracellular localization of PERK by cell fractionation using homogenates of murine liver and analyzed subcellular fractions constituted by the mitochondria, ER, MAMs, nucleus and cytosol. We found that PERK localized not only in the ER fraction and partially in the nuclear fraction as expected, but was also enriched in the MAM fraction, while it was poorly associated with the pure or crude mitochondrial fractions (Figure 7a).

We also extended this result to a human cell line and used a mitochondrial fractionation utilizing magnetic beads coated with a human anti-TOM22 antibody. PERK was found partially associated with the fraction of cell lysates containing crude mitochondria along with MAMs as contaminants (Figure 7b), as well as in the supernatant mainly consisting of unlabeled organelles and cell components. After trypsin treatment of the lysates, resulting in the isolation of a pure mitochondria fraction, PERK together with calnexin (another MAM component) were only detected in the supernatant (Figure 7b), thus indicating that PERK is part of a proteinaceous complex connecting the ER to the mitochondria.

We next reasoned that if the increased distance between ER and mitochondria due to PERK deficiency impairs the transfer of ROS-mediated effectors between these organelles, resulting in an attenuation of mitochondria-mediated apoptosis, then another cell line lacking a crucial component of the MAMs should display similar resistance against phoxER stress.

To test this hypothesis, we used MFN2-deficient MEFs. MEFs lacking MFN2 have a fragmented ER morphology ${ }^{28}$ and weaker ER-mitochondria interactions, to a degree similar to what is found in this study, which in turn reduces the efficiency of mitochondrial $\mathrm{Ca}^{2+}$ uptake. MFN2 ${ }^{-1-}$ cells displayed delayed kinetics of $\mathrm{CHOP}$ induction (Figure $5 \mathrm{c}$ ) and caspase-3 processing after phox-ER stress as compared with

Figure 6 PERK is required to maintain ER-mitochondrial contact sites and for phox-ER stress induced relocation of mitochondria to the ER. (A) Confocal analysis of untreated (Ctr) or phox-treated $\left(1 \mathrm{~J} / \mathrm{cm}^{2}\right)$ PERK ${ }^{+1+}$ and PERK ${ }^{-1-}$ MEFs. At the indicated time points, cells were fixed and co-stained for calreticulin (ER marker, red), TOM20 (mitochondrial marker, green) and nucleus (blue). Bars represent $20 \mu \mathrm{m}$. (B) Manders' colocalization analysis for data represented in (a-f). Graph represents mean \pm S.D. ( $n=3$, at least $25-30$ cells were analyzed per condition). (C) Confocal analysis of PERK ${ }^{+1+}$ and PERK ${ }^{-1-}$ MEFs transiently transfected with either empty vector (pcDNA) or a kinase-dead (PERK-K618A, left panel) or C-terminal deletion mutant (PERK $\triangle \mathrm{C}$, right panel) of PERK. Seventy two hours after transfection, cells were fixed and co-stained for calreticulin (ER marker, red), TOM20 (mitochondrial marker, green) and nucleus (blue). Bars represent $20 \mu \mathrm{m}$. (D) Western blot analysis of PERK K618A and PERK $\triangle$ C expression levels in PERK ${ }^{-1-}$ MEFs. Seventy two hours after transfection, whole cell lysates were immunoblotted for total PERK. Asterisk $\left({ }^{*}\right)$ indicates an unspecific band. (E) Manders' colocalization analysis for data represented in (A) (panels a and d) and (C). Graph represents mean \pm S.D. $(n=3$, at least $25-30$ cells were analyzed per condition). (f) Dissipation of mitochondrial membrane potential $\left(\Delta \psi_{\mathrm{m}}\right)$ following phox-ER stress in PERK ${ }^{+1+}, \mathrm{PERK}^{-1-}$ and PERK ${ }^{-1-}$ MEFs $72 \mathrm{~h}$ after transfection with either empty vector or a kinase-dead (PERK K618A) or C-terminal deletion (PERK $\Delta$ C) mutant of PERK. Cells were left untreated (Ctr) or phox-treated $\left(1.62 \mathrm{~J} / \mathrm{cm}^{2}\right)$. After $6 \mathrm{~h}$, cells were incubated with $2 \mathrm{nM}$ DiOC6(3) and the percentage of cells with reduced membrane potential $($ DiOC6(3) -$)$ was determined by flow cytometry. Note that the increased cell density used in this set of experiments attenuated the magnitude of $\Delta \psi_{\mathrm{m}}$ after phox-ER stress compared with that reported in Figure $5 \mathrm{~d}$. Graph represents the mean \pm S.D. of three independent experiments performed in duplicate. $\left(^{*}\right)$ indicates $P<0.05,\left({ }^{* *}\right) P<0.01$ and $\left({ }^{\star \star *}\right) P<0.001$ under Student's $t$-test 
a

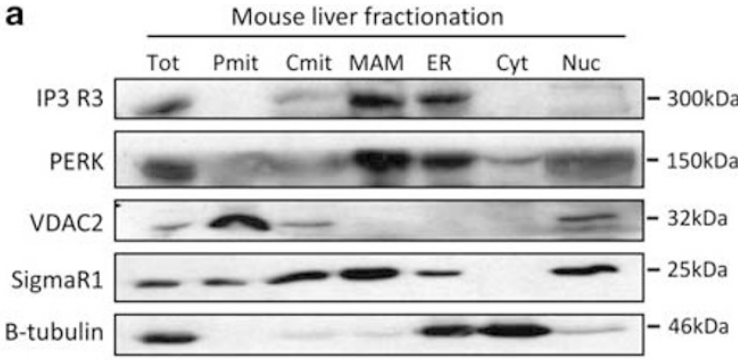

b

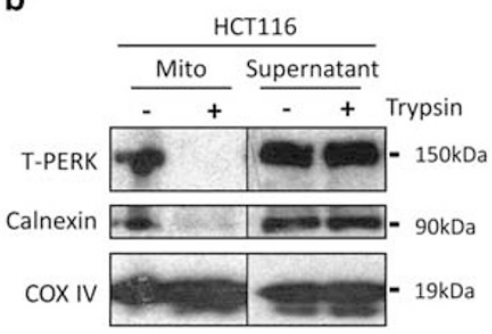

C
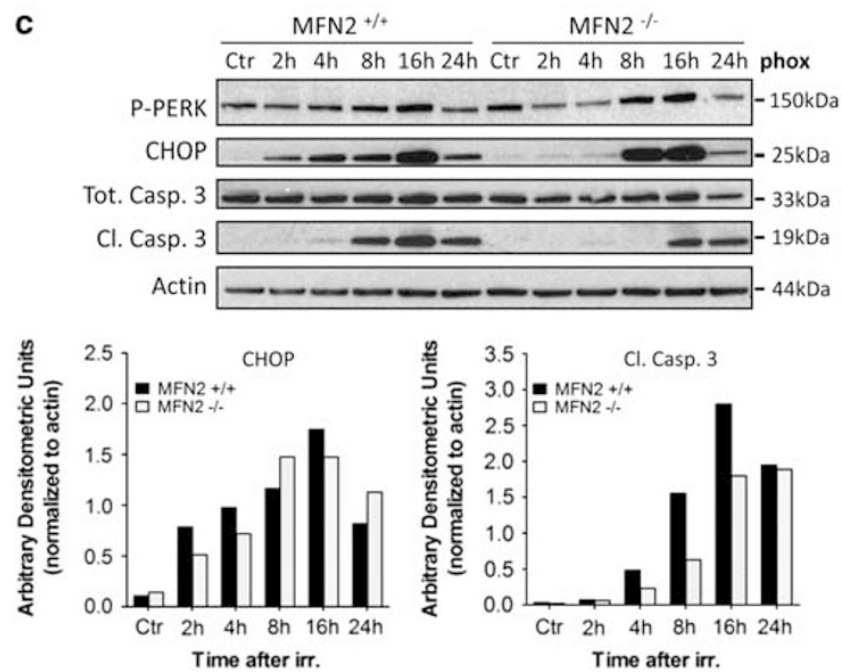

d
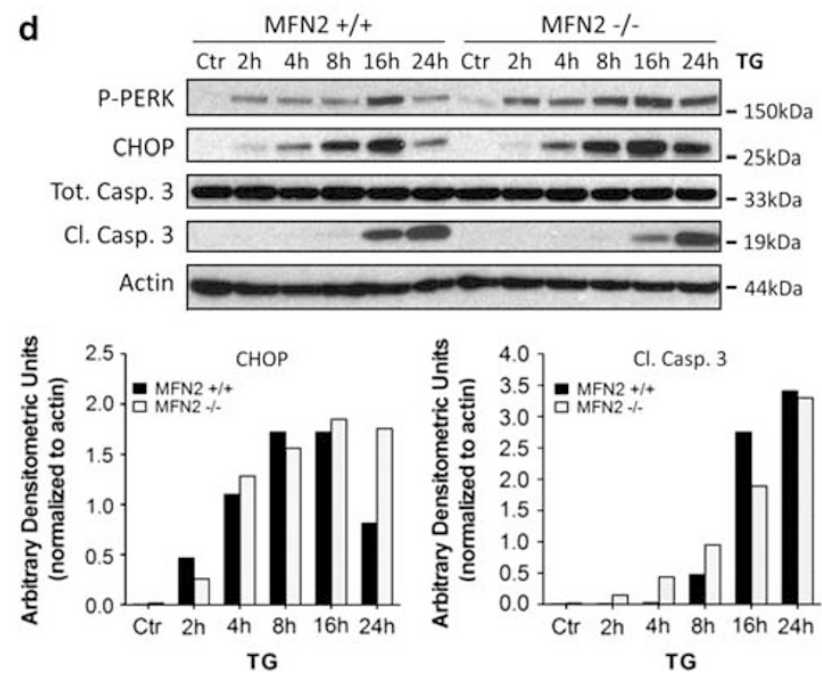

Figure 7 PERK is a component of the MAMs. (a) Western blot analysis of the indicated proteins obtained from subcellular fractionations of mouse hepatocytes. Tot, total cell lysate; Pmit, pure mitochondria; Cmit, crude mitochondria; MAM, mitochondria-associated ER membrane fraction; ER, endoplasmic reticulum fraction; Cyt, cytosolic fraction; Nuc, nuclear fraction. (b) Mitochondrial fractions were isolated from whole cell lysates from HCT116 cells by TOM22-coupled magnetic beads. Where indicated, cell lysates were pretreated with trypsin to cleave off MAMs. Mitochondrial fractions and supernatant were immunoblotted for the indicated proteins. Left and right parts are from the same membrane. After phox treatment (c) or TG $(2 \mu \mathrm{M})$ treatment (d) of MFN2 ${ }^{-1-}$ and MFN2 $2^{-1-}$ MEFs, whole cell lysates were made at the indicated time points and immunoblotted for the indicated proteins. Top: A representative experiment is shown. Bottom: Densitometric analysis for CHOP induction and caspase 3 cleavage. Mean values for four independent experiments are shown

their WT counterparts. Conversely, MNF2 deficiency did not notably affect caspase signaling and $\mathrm{CHOP}$ induction after TG treatment (Figure 7d).

These data highlight that (i) PERK is a novel component of the MAMs and (ii) perturbations of the ER/mitochondriacontact sites reduce the propagation of ROS signals to the neighboring mitochondria attenuating the onset of intrinsic apoptosis evoked by ROS-based ER stress (Figure 8).

\section{Discussion}

In this study utilizing a paradigm of ROS-based ER stress (i.e., phox-ER stress), we identified a novel function of PERK beyond its canonical role as ER stress sensor. We show that PERK is a novel molecular mediator of the ER-mitochondria contact sites, required to regulate inter-organellar cross-talk in ROS-induced cell death (Figure 8), a role which is not shared with other ER stress sensors, like IRE1.

The ER and mitochondria are physically and functionally connected through the MAMs. These specialized contact sites are crucial to regulate $\mathrm{Ca}^{2+}$ dynamics in response to $\mathrm{IP}_{3}$-mediated signaling or passive discharge of the $\mathrm{ER} \mathrm{Ca}^{2+}$ stores and accommodate lipid transfer between these two organelles, thereby ensuring spatiotemporal organization of cell signaling. ${ }^{11}$

We found that PERK is enriched at the ER face of the MAMs along with known MAM components, including the $\mathrm{IP}_{3} \mathrm{R} 3$ and the chaperones calnexin and Sigma Receptor $1 .{ }^{11}$ The novel function of PERK as regulator of the ER-mitochondria interface shown in this study appears to be independent of PERK's function as ER stress sensor and transcriptional regulator of redox homeostasis found in previous reports. ${ }^{29,30}$

PERK $^{+1+}$ and PERK ${ }^{-1-}$ cells in fact experience a similar initial burden of phox-mediated stress, yet loss of PERK results in a dramatic structural modification of the ER-mitochondria juxtapositions that prevents the propagation of ROS signaling to the apoptotic machinery. Consistent with this, only the early oxidation of cardiolipin, which precedes cytochrome $\mathrm{c}$ release and mitochondrial apoptosis following phox-ER stress, is significantly attenuated in $\mathrm{PERK}^{-1}$ - cells, whereas it is not affected by CHOP deficiency.

Furthermore, only the re-expression of a PERK kinasedead mutant in the PERK ${ }^{-1-}$ cells and not of a mutant lacking the cytoplasmic domain of PERK restored ER-mitochondria tethering and resensitized mitochondria to phox-ER stress.

The dispensability of PERK kinase activity for ERmitochondria tethering appears to exclude the possibility that 


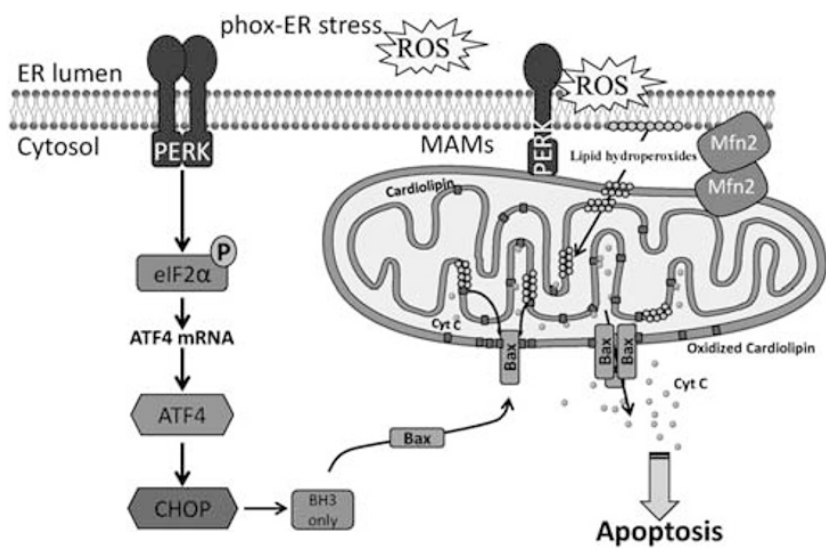

Figure 8 PERK has a dual role in ER stress signaling. PERK regulates photooxidative (phox)-ER stress induced apoptosis via two distinct mechanisms. On the one hand, canonical signaling via the PERK-eIF2 $\alpha$-ATF4 branch of the UPR induces the pro-apoptotic transcription factor $\mathrm{CHOP}$, which in turn mediates mitochondrial apoptosis through the upregulation of $\mathrm{BH} 3$ only proteins, like Bim, leading to Bax activation. On the other hand, PERK is required at the MAMs for tethering the ER to mitochondria, thereby promoting the rapid transfer of ROS signals, likely under the form of lipid hydroperoxides, from the ER to the mitochondria. As a result, the mitochondrial phospholipid cardiolipin is oxidized (and possibly externalized to the outer mitochondrial membrane) and cytochrome $c$ is released from the pool of mitochondria in close contact with the ER (where ROS are formed). Sustained cardiolipin oxidation may contribute to the insertion of Bax into the outer mitochondrial membrane inducing full blown mitochondrial apoptosis

the observed effects might be secondary to the induction of other MAM components like ERO1 $\alpha^{31,32}$ and the truncated variant of the SERCA pump $(\mathrm{S} 1 \mathrm{~T})^{33}$ by the PERK-ATF4/ CHOP pathway.

Thus, it is PERK's structural/scaffolding function at the MAMs, rather than its UPR function, which is crucial to transmit intrinsic apoptosis in response to ROS.

This notion is reinforced by the observation that MFN2 ${ }^{-1-}$ cells exposed to phox-ER stress display some of the characteristics of $\mathrm{PERK}^{-1-}$ cells, like delayed $\mathrm{CHOP}$ induction and caspase signaling. This is consistent with a proposed role for MFN2 in propagating oxidative stressmediated apoptosis in cardiomyocytes. ${ }^{34}$

Other recent studies have highlighted the relevance of ER-mitochondria juxtapositions in propagating apoptosis after pro-oxidant inducers of ER stress. Disturbing the ER-mitochondria juxtapositions by the expression of keratinbinding protein trichoplein/mitostatin has been shown to inhibit apoptosis following $\mathrm{H}_{2} \mathrm{O}_{2}$ exposure, while being ineffective against TG or other stimuli. ${ }^{35}$ Likewise, cells lacking the tumor suppressor promyelocytic leukemia, which also localizes at the MAMs, were more efficiently protected against agents causing ROS-dependent ER $\mathrm{Ca}^{2+}$ depletion, like $\mathrm{H}_{2} \mathrm{O}_{2}$ and menadione, rather than in response to etoposide or TG. ${ }^{36}$

Thus, these studies suggest that MAMs may provide specialized contact sites to transmit $\mathrm{Ca}^{2+}$ as well as ROSmediated signals to mitochondria following ROS-based ER stress.

An interesting possibility could involve trafficking of lipid hydroperoxides formed after phox treatment ${ }^{8}$ through the MAMs to the juxtaposed mitochondria where, along with
$\mathrm{Ca}^{2+}$ signals, they could sensitize these mitochondria to intrinsic apoptosis.

Consistently, accumulation of GM1 ganglioside within the MAMs influenced $\mathrm{Ca}^{2+}$ flux between the ER and the mitochondria, which in turn promoted ER stress-induced mitochondria-mediated apoptosis. ${ }^{6}$

Irrespective of the molecular nature of these signaling components, the unique role of PERK at the ER-mitochondria contact sites discovered here suggests that in pathological conditions linked to loss of PERK, mitochondria may be less responsive to agents that mobilize $\mathrm{ER} \mathrm{Ca}^{2+}$ through a ROSdependent process. Moreover, whether PERK governs the pre-apoptotic emission of damage-associated molecular patterns from the dying cancer cells following phox-ER stress ${ }^{9}$ through UPR-independent mechanisms that require its role at the MAMs, is an intriguing perspective that deserves to be further investigated.

\section{Materials and Methods}

Reagents. Hypericin was prepared and stored as reported in previous work. ${ }^{8}$ Thapsigargin was from Enzo Life Sciences (Farmingdale, NY, USA), Fura-2AM from TEFlabs (Austin, TX, USA), DiOC6(3) ER-Tracker Blue-White DPX and nonyl acridine orange (NAO) from Life Technologies (Carlsbad, CA, USA) and ATP from Roche Applied Science (Indianapolis, IN, USA). IP 3 was from Sigma-Aldrich (St. Louis, MO, USA). Antibodies against total and phospho PERK, total and phospho elF2 $\alpha$, total and phospho-JNK1\&2, GRP78, total and active caspase 3 (cleaved fragments only), caspase 9, PARP, Calnexin, XBP1s and COXIV were from Cell Signaling Technology (Danvers, MA, USA). Actin, tubulin and SigmaR1 antibodies were from Sigma-Aldrich. Anti-cytochrome $\mathrm{C}$ antibodies were from $\mathrm{BD}$ Pharmingen (San Jose, CA, USA), Antibodies against TOM20, Calreticulin (CRT), MFN2 and VDAC-2 were from Abcam (Cambridge, UK) and anti-IP ${ }_{3} R 3$ antibody was from BD Biosciences (Franklin Lakes, NJ, USA).

Western blotting. Preparation of cell lysates, determination of protein concentration and sample preparation for western blotting were described in previous work. ${ }^{37}$ Samples were processed on the Criterion system (Bio-Rad Laboratories, Hercules, CA, USA) on a 4-12\% Bis-TRIS gel and Protran $2 \mu \mathrm{m}$ pored nitrocellulose paper (Perkin-Elmer, Wellesley, MA, USA). Enhanced chemiluminescence was used as WB detection system. Image $\mathrm{J}(\mathrm{NIH}$, Washington, DC, USA) was used for quantification of immunoblots according to http:// lukemiller.org/index.php/2010/11/analyzing-gels-and-western-blots-with-image-j/.

Cell culture. Simian virus 40 (SV40)-immortalized MEFs deficient for PERK, CHOP and IRE1, KRAS-transformed PERK ${ }^{+1+}$ and PERK ${ }^{-1-}$ MEFs and CT26 cells were maintained in Dulbecco's modified Eagle's medium containing $4.5 \mathrm{~g} / \mathrm{l}$ glucose and $0.11 \mathrm{~g} / \mathrm{l}$ sodium pyruvate and supplemented with $2 \mathrm{mM}$ glutamine, $100 \mathrm{units} / \mathrm{ml}$ penicillin, $100 \mu \mathrm{g} / \mathrm{ml}$ streptomycin and $10 \%$ fetal bovine serum (FBS) (AA medium). MDA-MB468 cells were maintained in DMEM supplemented with $5 \% \mathrm{FBS}, 0.01 \mathrm{mg} / \mathrm{ml}$ insulin and antibiotics.

phox treatment. Cells (except those that were electroporated, see further) were plated $24 \mathrm{~h}$ before treatment to obtain $\pm 70 \%$ confluency on the day of phox treatment. Before irradiation, MEFs were incubated with $150 \mathrm{nM}$ hypericin (MFN2 MEFs) or $200 \mathrm{nM}$ hypericin (PERK, CHOP and IRE1 MEFs) for $2 \mathrm{~h}$ in FBS-free DMEM and 10\% FBS (Thermo Scientific HyClone, Logan, UT, USA) was reconstituted after irradiation. Irradiation was performed as described before ${ }^{8}$ or on a low pressure sodium lamp (SOX-E36W; Philips, Eindhoven, The Netherlands) using a fluency rate of $15 \mathrm{~mW} / \mathrm{cm}^{2}$ as measured with an IL 1400 radiometer (International light, Newburyport, MA, USA) with a total fluency of $1.49 \mathrm{~J} / \mathrm{cm}^{2}$ unless stated differently. CT26 and MDA-MB468 cells were incubated with $150 \mathrm{nM}$ hypericin in AA medium. After $16 \mathrm{~h}$, cells were irradiated with total fluency of $1.49 \mathrm{~J} /$ $\mathrm{cm}^{2}$ (MDA) or $1.89 \mathrm{~J} / \mathrm{cm}^{2}$ (CT26). All controls define cells incubated with hypericin but not exposed to light irradiation.

Transfection/electroporation. PERK ${ }^{-1-}$ cells were transiently transfected with either empty vector (pcDNA3.1; Life Technologies) or plasmid 
encoding PERK-K618 $\mathrm{A}^{27}$ (Addgene plasmid 21815) or PERK $\Delta \mathrm{C}$ (a C-terminal deletion-mutant lacking amino acids 582-1081; Addgene plasmid 21816) together with a cytosolic GFP-encoding plasmid (pGFPmax; Lonza, Walkersville, MD, USA) using a Neon transfection system according to manufacturer's protocol (Life Technologies). Twenty hours after electroporation, surviving cells were replated to culture dishes/slides and allowed to grow for an additional $48 \mathrm{~h}$ to obtain optimal expression levels of the PERK mutants before fluorescence microscopy analysis or phox treatment. Note that this experimental set-up results in $\pm 90 \%$ cell confluency on the day of phox treatment. Higher confluency is a factor reported in previous studies to account for increased cellular protection against phox treatment. $^{38}$

RT-PCR, PCR and QPCR. Total RNA was extracted using the PureLink RNA Mini Kit (Life Technologies). Total RNA $(2 \mu \mathrm{g})$ was reverse-transcribed to cDNA using the Superscript III reverse transcription kit (Life Technologies). For determination of Xbp1 splicing, the cDNA product was subjected to 25-35 cycles of PCR using the forward primer $5^{\prime}$-GAACCAGGAGTTAAGAACACG- $3^{\prime}$ and reverse primer $5^{\prime}$-AGGCAACAGTGTCAGAGTCC-3' specific for mouse XBP-1 were used. GAPDH (forward: ACCACAGTCCATGCCATG; reverse: TCCACCACCCTGTTGCTG) was used as an endogenous control. Products were separated by electrophoresis through a $2 \%$ agarose gel and visualized by ethidium bromide staining. For quantitative real-time PCR to determine the induction of UPR target genes, cDNA products were mixed with Brilliant III ultra-fast master mix (Agilent Technologies, Santa Clara, CA, USA) and $20 \times$ TaqMan Gene Expression Assays (Applied Biosystems, Carlsbad, CA, USA/Integrated DNA Technologies, Coralville, IA, USA) and subjected to 40 cycles of PCR in a 7500 StepOnePlus instrument (Applied Biosystems). The relative quantity of each target transcript was normalized to GAPDH and relative expression was evaluated with $\Delta \Delta \mathrm{C}_{\mathrm{T}}$ method. Primer sets for GRP78, P5, ER01L, ER01Lb, Derl2, Hrd1 and GRP94 are summarized in Table 1 of Supplementary Figure 7. Primer sets for P58ipk, ERP72, CHOP and EDEM1 are summarized in Table 2 of Supplementary Figure 7.

Fluorescence microscopy. Cells were grown and treated on chambered coverglasses (Nunc, Roskilde, Denmark). Cells were fixed with $4 \%$ p-formaldehyde (Sigma-Aldrich), permeabilized with $0.1 \%$ Triton X-100 (v/v in PBS), blocked with $10 \% \mathrm{BSA}$ and incubated with stated primary and secondary antibodies (Dylight680b (Thermo Fisher Scientific; Rockford, IL, USA) and AlexaFluor488 (Life Technologies)) in blocking buffer. Finally, nuclear DAPI staining was performed $(1 \mathrm{mg} / \mathrm{ml}$, Life Technologies) and cells were mounted using Prolong Gold antifade reagent (Life Technologies). Confocal fluorescence images were acquired using a Nikon A1R confocal unit mounted on a Ti2000 inverted microscope controlled by NIS elements acquisition software (Nikon Instruments Inc., Melville, NY, USA) using a Plan APO VC $\times 601.4$ NA oil immersion objective. Images were analyzed using ImageJ (WS Rasbaud, ImageJ, National Institute of Health, Bethesda, MD, USA; http://rsb.info.nih.gov/ij/). Colocalization between the ER (calreticulin) and mitochondria (TOM20) was quantified by calculating Mander's coefficients using JACoP plugin for ImageJ.

Transmission Electron Microscopy. Transmission Electron Microscopy (TEM) was performed as described in Buytaert et al. ${ }^{8}$ In short, cells were fixed in $0.1 \mathrm{M}$ sodium cacodylate-buffered $(\mathrm{pH} 7.4) 2.5 \%$ glutaraldehyde solution and postfixed in $0.1 \mathrm{M}$ sodium cacodylate-buffered $(\mathrm{pH} 7.4) 1 \% \mathrm{OsO}_{4}$ solution. After dehydration in an ethanol gradient, samples were incubated with propylenoxid, impregnated with a mixture of propylenoxid/LX-112 (1:1; Ladd Research Industries, Williston, VT, USA) and embedded in LX-112. Ultrathin sections were stained with uranyl acetate and lead citrate. Sections were examined in a Philips CM10 microscope (Philips Research, Eindhoven, The Netherlands) at $80 \mathrm{kV}$.

ROS measurements. At the desired time points, control cells and treated cells were trypsinized and resuspended in Phenol Red-free DMEM supplemented with $7.5 \mu \mathrm{M}$ of 5 -(and-6)-chloromethyl-2', $7^{\prime}$-dichlorodihydrofluorescein diacetate acetyl ester (CM- $\left.\mathrm{H}_{2} \mathrm{DCF}-\mathrm{DA}\right)$ (Life Technologies). After incubation, cells were washed once with and resuspended in ice-cold PBS. Samples were immediately analyzed with a BD FACSCanto II flow cytometer (FITC channel; 515-545 nm) with BD FACSdiva software (BD Biosciences).

Cell death and clonogenic assays. Analysis of apoptosis was performed by western blot analysis for cytochrome $c$ release, caspase 9 and caspase 3 processing and PARP cleavage. Analysis of cell death was performed with Trypan Blue Exclusion Assay as described in Buytaert et al. ${ }^{8}$ Alternatively, to determine the amount of cell death, plasma membrane permeabilization was analyzed by estimating the amounts of LDH released into the culture media using LDH Cytotoxicity Detection Kit (Clontech, Mountain View, CA, USA) according to manufacturer's instructions. For clonogenic assays, KRAS-transduced WT and PERK $^{-1-}$ MEFs were treated with hypericin or TG and subsequently replated at low density to $10 \mathrm{~cm}$ dishes. After 10 days of incubation, cells were stained with $1 \%$ methylene blue in methanol and colonies of at least 50 cells were counted.

Mitochondrial membrane potential and cardiolipin peroxidation. At the desired time points, loss of mitochondrial membrane potential and cardiolipin oxidation were determined by loading the cells with $2 \mathrm{nM}$ DiOC6(3) or $10 \mathrm{nM} \mathrm{NAO}$, respectively. After trypsinization, cells were resuspended in icecold PBS and samples were immediately analyzed with a BD FACSCanto II flow cytometer (FITC channel; 515-545 nm) with BD FACSdiva software (BD Biosciences). Results were further processed with FlowJo software (Tree Star, Inc., Ashland, OR, USA).

$\mathrm{Ca}^{2+}$ experiments. Cytosolic $\mathrm{Ca}^{2+}$ signals were measured by loading the cells with Fura-2AM (at a final concentration of $1.25 \mu \mathrm{M}$ ) for $30 \mathrm{~min}$ at room temperature in modified KREBS buffer containing $135 \mathrm{mM} \mathrm{NaCl}, 5.9 \mathrm{mM} \mathrm{KCl}$, $1.2 \mathrm{mM} \mathrm{MgCl}_{2}, 11.6 \mathrm{mM}$ Hepes (pH 7.3), $11.5 \mathrm{mM}$ glucose and $1.5 \mathrm{mM} \mathrm{Ca}^{2+}$. Cells were then incubated for at least $30 \mathrm{~min}$ in the absence of Fura-2AM allowing its de-esterification. Ratiometric $\mathrm{Ca}^{2+}$ signals were obtained by acquiring emission at $510 \mathrm{~nm}$ while exciting at 340 and $380 \mathrm{~nm}$ with an automated bench top microplate reader (FlexStation 3; Molecular Devices, Sunnyvale, CA, USA). Unidirectional ${ }^{45} \mathrm{Ca}^{2+}$-flux analysis was performed as described in Missiaen et al..$^{39} \mathrm{Ca}^{2+}$ entry experiments were performed as described in Decuypere et al. ${ }^{40}$

Subcellular fractionation. Liver from $\mathrm{C} 57 / \mathrm{B} \mid 6$ mice was excised, extensively washed with PBS, minced, resuspended in isolation buffer $(50 \mathrm{mM}$ Tris $\mathrm{pH}=7.4,150 \mathrm{mM} \mathrm{NaCl}, 1 \mathrm{mM}$ EGTA and $250 \mathrm{mM}$ sucrose) and homogenized in a glass Dounce homogenizer with a Teflon pestle (five strokes). The homogenate was spun at $800 \times g$ for $10 \mathrm{~min}$ to remove entire cells and nuclei; the supernatant was recovered and further centrifuged for $10 \mathrm{~min}$ at $8000 \times \mathrm{g}$. The resulting pellet (crude mitochondrial fraction) was collected while the supernatant was further spun for $30 \mathrm{~min}$ at $100000 \times \mathrm{g}$. The resulting pellet (LM fraction) and supernatant (cytosolic fraction) were spun again at $100000 \times g$ to further purify the fractions. The crude mitochondrial fraction was further purified by centrifuging twice at $8000 \times g$ for $10 \mathrm{~min}$. The obtained pellet was purified by centrifugation at $95000 \times g$ for $30 \mathrm{~min}$ on a $30 \%$ Percoll density gradient in IB. The low-density band (denoted as MAM fraction) and the high-density band (denoted as pure mitochondria) were collected. The obtained mitochondrial layer was washed free of Percoll and resuspended in IB. For SDS-PAGE analysis, $20 \mu \mathrm{g}$ of proteins from each fraction was loaded on 10\% SDS-polyacrylamide gels and blotted onto a nitrocellulose membrane.

Mitochondrial isolation. Isolation of mitochondria from HCT116 whole cell lysates was performed with the MACS Mitochondrial Isolation Kit according to manufacturer's protocol (Miltenyi Biotec GmbH, Bergisch Gladbach, Germany). MAM components were eluted by trypsin $\left(2 \mathrm{~min}, 40 \mu \mathrm{g} / \mathrm{ml}, 37^{\circ} \mathrm{C}\right)$.

Statistical analysis. Data are presented either in fold changes or in percentages with mean \pm S.D. (standard deviation) or S.E.M. (standard error of mean) as indicated in figure legends. All statistical analyses were performed using either Prism software (GraphPad Software, La Jolla, CA, USA) or GraphPad QuickCalcs online software (http://www.graphpad.com/quickcalcs/index.cfm). Unpaired Student's t-test was used for statistical analysis.

\section{Conflict of Interest}

The authors declare no conflict of interest.

Acknowledgements. This work is supported by GOA grant of the KU Leuven and by F.W.O grant G.0492.05 to PA, G.0788.11N, and OTSRT1/10/44 to GB. This paper presents research results of the IAP6/18 and IAP6/28, funded by the Interuniversity Attraction Poles Programme, initiated by the Belgian State, Science 
Policy Office. NR is a post-doctoral fellow supported by F.R.S-FNRS (grant F/5/4/5$\mathrm{MCF} / \mathrm{KP}$ ). J-PD is a recipient of a PhD Fellowship from the Agency for Innovation of Science and Technology (IWT). We thank Dr. R Kaufman (University of Michigan Medical Center, Ann Arbor, MI, USA) for the WT, PERK ${ }^{-1-}, \mathrm{CHOP}^{-1-}$ and IRE $1^{-1-}$ fibroblasts, Dr. A Diehl (Abramson Family Cancer Research Institute Philadelphia, USA) for the PERK-shRNA transduced MDA-MB468 cells, Dr. D Krysko (University of Ghent, Belgium) for the PERK-shRNA CT26 cells and Dr. W Martinet (University of Antwerpen, Belgium) for the electron microscopy analysis. We thank Professor M Fransen for the use of the Neon Electroporation equipment. We acknowledge Dr. S Bontems for excellent support in transforming MEFs with KRAS, Tomas Luyten for excellent support in the $\mathrm{Ca}^{2+}$ experiments, Veerle Daniels for help with MACs isolation, and Jan Piessens and Kristine Rillaerts for skillful technical support. Nikon confocal microscope was acquired through a Hercules Type 1 AKUL/09/037 to W Annaert.

1. Malhotra JD, Kaufman RJ. The endoplasmic reticulum and the unfolded protein response. Semin Cell Dev Biol 2007; 18: 716-731.

2. Tabas I, Ron D. Integrating the mechanisms of apoptosis induced by endoplasmic reticulum stress. Nat Cell Biol 2011; 13: 184-190.

3. Verfaillie $T$, Garg $A D$, Agostinis $P$. Targeting ER stress induced apoptosis and inflammation in cancer. Cancer Lett 2010; e-pub ahead of print 21 August 2010; doi: 10.1016/ j.canlet.2010.07.016.

4. Malhotra JD, Kaufman RJ. Endoplasmic reticulum stress and oxidative stress: a vicious cycle or a double-edged sword? Antioxid Redox Signal 2007; 9: 2277-2293.

5. Pinton P, Giorgi C, Pandolfi PP. The role of PML in the control of apoptotic cell fate: a new key player at ER-mitochondria sites. Cell Death Differ 2011; 18: 1450-1456.

6. Sano R, Annunziata I, Patterson A, Moshiach S, Gomero E, Opferman J et al. GM1-ganglioside accumulation at the mitochondria-associated ER membranes links ER stress to $\mathrm{Ca}(2+)$-dependent mitochondrial apoptosis. Mol Cell 2009; 36: 500-511.

7. Agostinis P, Berg K, Cengel KA, Foster TH, Girotti AW, Gollnick SO et al. Photodynamic therapy of cancer: an update. CA Cancer J Clin 2011; 61: 250-281.

8. Buytaert E, Callewaert G, Hendrickx N, Scorrano L, Hartmann D, Missiaen L et al. Role of endoplasmic reticulum depletion and multidomain proapoptotic BAX and BAK proteins in shaping cell death after hypericin-mediated photodynamic therapy. FASEB J 2006; 20: 756-758.

9. Garg AD, Krysko DV, Verfaillie T, Kaczmarek A, Ferreira GB, Marysael T et al. A novel pathway combining calreticulin exposure and ATP secretion in immunogenic cancer cell death. EMBO J 2012; 31: 1062-1079.

10. Buytaert E, Matroule JY, Durinck S, Close P, Kocanova S, Vandenheede JR et al. Molecular effectors and modulators of hypericin-mediated cell death in bladder cance cells. Oncogene 2008; 27: 1916-1929.

11. Hayashi T, Rizzuto R, Hajnoczky G, Su TP. MAM: more than just a housekeeper. Trends Cell Biol 2009; 19: 81-88

12. Yamamoto K, Sato T, Matsui T, Sato M, Okada T, Yoshida $\mathrm{H}$ et al. Transcriptional induction of mammalian ER quality control proteins is mediated by single or combined action of ATF6alpha and XBP1. Dev Cell 2007; 13: 365-376.

13. Harding HP, Zhang Y, Zeng H, Novoa I, Lu PD, Calfon M et al. An integrated stress response regulates amino acid metabolism and resistance to oxidative stress. $\mathrm{Mol} \mathrm{Cell}$ 2003; 11: 619-633.

14. Yamaguchi $Y$, Larkin D, Lara-Lemus R, Ramos-Castaneda J, Liu M, Arvan P. Endoplasmic reticulum (ER) chaperone regulation and survival of cells compensating for deficiency in the ER stress response kinase, PERK. J Biol Chem 2008; 283 17020-17029.

15. Redondo PC, Salido GM, Rosado JA, Pariente JA. Effect of hydrogen peroxide on Ca2 + mobilisation in human platelets through sulphydryl oxidation dependent and independent mechanisms. Biochem Pharmacol 2004; 67: 491-502.

16. Wei H, Li Z, Hu S, Chen X, Cong X. Apoptosis of mesenchymal stem cells induced by hydrogen peroxide concerns both endoplasmic reticulum stress and mitochondria death pathway through regulation of caspases, p38 and JNK. J Cell Biochem 2010; 111: 967-978.

17. Harding HP, Zhang $Y$, Bertolotti $A$, Zeng $H$, Ron D. Perk is essential for translationa regulation and cell survival during the unfolded protein response. Mol Cell 2000; 5 : 897-904.
18. Novosyadlyy R, Kurshan N, Lann D, Vijayakumar A, Yakar S, LeRoith D. Insulin-like growth factor-I protects cells from ER stress-induced apoptosis via enhancement of the adaptive capacity of endoplasmic reticulum. Cell Death Differ 2008; 15: 1304-1317.

19. Huang G, Yao J, Zeng W, Mizuno Y, Kamm KE, Stull JT et al. ER stress disrupts Ca2 + signaling complexes and $\mathrm{Ca} 2+$ regulation in secretory and muscle cells from PERKknockout mice. J Cell Sci 2006; 119(Part 1): 153-161.

20. Hogan PG, Lewis RS, Rao A. Molecular basis of calcium signaling in lymphocytes: STIM and ORAI. Annu Rev Immunol 2010; 28: 491-533.

21. Hendrickx N, Dewaele M, Buytaert E, Marsboom G, Janssens S, Van Boven M et al. Targeted inhibition of p38alpha MAPK suppresses tumor-associated endothelial cell migration in response to hypericin-based photodynamic therapy. Biochem Biophys Res Commun 2005; 337: 928-935.

22. Agarwal ML, Larkin HE, Zaidi SI, Mukhtar H, Oleinick NL. Phospholipase activation triggers apoptosis in photosensitized mouse lymphoma cells. Cancer Res 1993; 53: 5897-5902.

23. Orrenius S, Zhivotovsky B. Cardiolipin oxidation sets cytochrome c free. Nat Chem Biol 2005; 1: 188-189.

24. Korytowski W, Basova LV, Pilat A, Kernstock RM, Girotti AW. Permeabilization of the mitochondrial outer membrane by Bax/truncated Bid (tBid) proteins as sensitized by cardiolipin hydroperoxide translocation: mechanistic implications for the intrinsic pathway of oxidative apoptosis. J Biol Chem 2011; 286: 26334-26343.

25. Rizzuto R, Pinton P, Brini M, Chiesa A, Filippin L, Pozzan T. Mitochondria as biosensors of calcium microdomains. Cell Calcium 1999; 26: 193-199.

26. Brough D, Schell MJ, Irvine RF. Agonist-induced regulation of mitochondrial and endoplasmic reticulum motility. Biochem J 2005; 392(Part 2): 291-297.

27. Harding HP, Zhang Y, Ron D. Protein translation and folding are coupled by an endoplasmic-reticulum-resident kinase. Nature 1999: 397: 271-274.

28. de Brito OM, Scorrano L. Mitofusin 2 tethers endoplasmic reticulum to mitochondria. Nature 2008; 456: 605-610.

29. Cullinan SB, Zhang D, Hannink M, Arvisais E, Kaufman RJ, Diehl JA. Nrf2 is a direct PERK substrate and effector of PERK-dependent cell survival. Mol Cell Biol 2003; 23: 7198-7209.

30. Bobrovnikova-Marjon E, Grigoriadou C, Pytel D, Zhang F, Ye J, Koumenis C et al. PERK promotes cancer cell proliferation and tumor growth by limiting oxidative DNA damage. Oncogene 2010; 29: 3881-3895.

31. Gilady SY, Bui M, Lynes EM, Benson MD, Watts R, Vance JE et al. Ero1alpha requires oxidizing and normoxic conditions to localize to the mitochondria-associated membrane (MAM). Cell Stress Chaperones 2010; 15: 619-629.

32. Anelli T, Bergamelli L, Margittai E, Rimessi A, Fagioli C, Malgaroli A et al. Ero1alpha regulates $\mathrm{Ca}(2+)$ fluxes at the endoplasmic reticulum-mitochondria interface (MAM). Antioxid Redox Signal 2012; 15: 1077-1087.

33. Chami M, Oules B, Szabadkai G, Tacine R, Rizzuto R, Paterlini-Brechot P. Role of SERCA1 truncated isoform in the proapoptotic calcium transfer from ER to mitochondria during ER stress. Mol Cell 2008; 32: 641-651.

34. Papanicolaou KN, Khairallah RJ, Ngoh GA, Chikando A, Luptak I, O'Shea KM et al. Mitofusin-2 maintains mitochondrial structure and contributes to stress-induced permeability transition in cardiac myocytes. Mol Cell Biol 2011; 31: 1309-1328.

35. Cerqua C, Anesti V, Pyakurel A, Liu D, Naon D, Wiche G et al. Trichoplein/mitostatin regulates endoplasmic reticulum-mitochondria juxtaposition. EMBO Rep 2010; 11: 854-860.

36. Giorgi C, Ito K, Lin HK, Santangelo C, Wieckowski MR, Lebiedzinska M et al. PML regulates apoptosis at endoplasmic reticulum by modulating calcium release. Science 2010; 330: 1247-1251.

37. Vantieghem A, Assefa Z, Vandenabeele P, Declercq W, Courtois S, Vandenheede JR et al. Hypericin-induced photosensitization of HeLa cells leads to apoptosis or necrosis. Involvement of cytochrome $\mathrm{c}$ and procaspase-3 activation in the mechanism of apoptosis. FEBS Lett 1998; 440: 19-24.

38. Delaey EM, Vandenbogaerde AL, Agostinis P, De Witte PA. Confluence dependent resistance to photo-activated hypericin in HeLa cells. Int J Oncol 1999; 14: 759-763.

39. Missiaen L, Parys JB, Weidema AF, Sipma H, Vanlingen S, De Smet P et al. The bellshaped $\mathrm{Ca} 2+$ dependence of the inositol 1,4,5-trisphosphate-induced $\mathrm{Ca} 2+$ release is modulated by Ca2 +/calmodulin. J Biol Chem 1999; 274: 13748-13751.

40. Decuypere JP, Monaco G, Bultynck G, Missiaen L, De Smedt H, Parys JB. The IP(3) receptor-mitochondria connection in apoptosis and autophagy. Biochim Biophys Acta 2011; 1813: 1003-1013. 